\title{
Effectiveness of Biofeedback in Improving Heart Rate Variability Recovery Following Stress
}

Amanda L. Wheat

West Virginia University

Follow this and additional works at: https://researchrepository.wvu.edu/etd

\section{Recommended Citation}

Wheat, Amanda L., "Effectiveness of Biofeedback in Improving Heart Rate Variability Recovery Following Stress" (2012). Graduate Theses, Dissertations, and Problem Reports. 476.

https://researchrepository.wvu.edu/etd/476

This Dissertation is protected by copyright and/or related rights. It has been brought to you by the The Research Repository @ WVU with permission from the rights-holder(s). You are free to use this Dissertation in any way that is permitted by the copyright and related rights legislation that applies to your use. For other uses you must obtain permission from the rights-holder(s) directly, unless additional rights are indicated by a Creative Commons license in the record and/ or on the work itself. This Dissertation has been accepted for inclusion in WVU Graduate Theses, Dissertations, and Problem Reports collection by an authorized administrator of The Research Repository @ WVU.

For more information, please contact researchrepository@mail.wvu.edu. 
Effectiveness of Biofeedback in Improving Heart Rate Variability

Recovery Following Stress

Amanda L. Wheat

\begin{abstract}
Dissertation submitted to the Eberly College of Arts and Sciences

at West Virginia University

in partial fulfillment of the requirements

for the degree of
\end{abstract}

Doctor of Philosophy

in

Psychology

Kevin Larkin, Ph.D., Chair

Amy Gentzler, Ph.D.

Lisa Kimmerer, Ph.D.

Tracy Morris, Ph.D.

Susannah Poe, Ed.D.

Department of Psychology

Morgantown, West Virginia

2012

Keywords: Heart Rate Variability, Biofeedback, Stress 


\section{Abstract \\ Effectiveness of Biofeedback in Improving Heart Rate Variability \\ Recovery Following Stress}

\section{Amanda L. Wheat}

The current study was designed to examine the efficacy of heart rate variability (HRV) biofeedback using emWave, a specific biofeedback device available to the public, and to determine whether such treatment affects phasic physiological responses to stress. Twenty-seven individuals (4 males, 23 females), aged $18-30$ years $(M=22.54$ years, $S D=3.82)$ participated in the current study. Thirteen individuals were randomly assigned to a control group and received no intervention, whereas the remaining 14 participants underwent 4-8 sessions of emWave intervention. All participants attended pre-treatment and post-treatment assessment sessions during which a stressor protocol was administered; physiological data were collected during rest, during stress, and following stress. EmWave treatment did not confer changes in resting, or tonic, measures of HRV. However, participants who received emWave treatment exhibited higher parasympathetic responses (i.e., pNN50) during stress at the post-treatment session compared to their control counterparts. Significant group differences in HRV were not evident during the post-stress recovery period. Additionally, no treatment effects were evident on selfreported measures of stress, psychological symptoms, or affect administered during the pre- and post-treatment assessment visits. Overall, results from the current study, combined with previous literature, suggest that the emWave may confer some limited treatment effects by increasing HRV in response to stress. Additional development and testing of the emWave treatment protocol is necessary before it can be recommended for regular use in clinical settings, including the determination of what physiological changes occur during HRV biofeedback that contribute to any clinical effects that may occur with the emWave treatment. 


\section{Table of Contents}

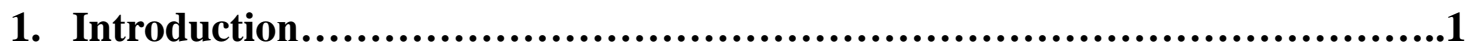

1.1. Heart Rate Variability Biofeedback......................................6

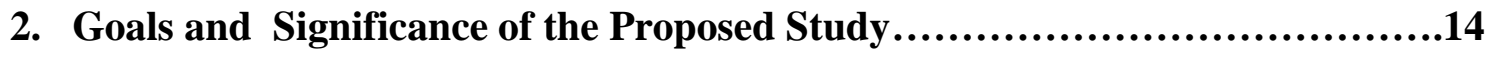

3. Methods.....................................................................15

3.1. Study Personnel and Timeline............................................15

3.2. Participants...............................................................16

3.3. Experimental and Analytical Design.......................................17

3.4. Measures...................................................................17

3.4.1. Demographics.......................................................17

3.4.2. Heart Rate Variability ...............................................18

3.4.3. Self-report Measures...............................................18

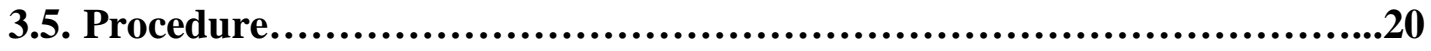

3.5.1. Stress Reactivity Protocol.................................................20

3.5.2. Treatment Protocol...............................................21

3.5.3. Post-treatment Assessment.........................................23

4. Results...................................................................24

4.1. Data Reduction and Preparation for Analyses...........................24

4.1.1. Self-report Outcomes................................................24

4.1.2. Physiological Outcomes............................................25

4.2. Preliminary Analyses......................................................26

4.2.1. Group Differences in Demographic Variables.......................26

4.2.2. Potential Covariates...............................................26 
4.2.3. Evidence of Stress Reactivity to Tasks.............................27

4.2.4. Tasks Differences.................................................27

4.2.5. Heart Rate Variability and emWave Performance During Treatment Sessions.............................................................28

4.2.6. Home Practice of Treatment......................................29

4.3. Primary Analyses..........................................................30

4.3.1. Physiological Outcomes............................................30

4.3.1.1. Tonic Measures of Heart Rate Variability.......................30

4.3.1.2. Phasic Heart Rate Variability Reactivity to Stress..............31

4.3.1.3. Heart Rate Variability Recovery from Stress....................31

4.3.1.4. Exploratory Analyses of Treatment Responders.................32

4.3.2. Supplementary Self-report Measures..............................32

4.3.2.1. Multiple Affect Adjective Checklist-Revised.....................32

4.3.2.2. Perceived Stress Scale...........................................33

4.3.2.3. Brief Symptom Inventory..................................33

5. Discussion.....................................................................33

5.1. Effects of emWave on Physiological Measures............................34

5.1.1. Tonic Heart Rate Variability....................................35

5.1.2. Phasic Heart Rate Variability......................................37

5.1.3. Heart Rate Variability Recovery..................................39

5.2. Effects of emWave on Affective Responses to Stress......................43

5.3. Effects of emWave on Stress and Psychological Symptom Distress........44

5.4. Study Limitations.......................................................46 
5.5. Future Directions......................................................50

6. Conclusions...............................................................52

7. References.........................................................................54

8. Appendices..................................................................64 


\section{Introduction}

Knowledge of interplay between physiological and psychological phenomena, and how those phenomena might be manipulated to promote health, have been persistent areas of growth in both medical and psychological fields of study since the inception of psychophysiology and behavioral medicine. For example, the last three decades have encapsulated progress discerning how various physiological and psychological factors affect each other, such as the interplay between amygdala activity and emotion (e.g., Phelps, 2006), cortisol and stress (e.g., Taylor, Burklund, Eisenberger, Lehman, Hilmert, Lieberman, 2008), cardiovascular reactivity and fear (e.g., Lerner, Dahl, Hariri, \& Taylor, 2007), and dopamine and temperament (e.g., Schmidt, Fox, Perez-Edgar, \& Hamer, 2009), among many others. Heart rate variability (HRV) is one of the newest physiological parameters that have gained attention in such a context (e.g., Shea, Kamath, Fleming, Streiner, Redmond, \& Steiner, 2008). In brief, HRV refers to oscillations in heart rate that occur naturally. Such fluctuations can be seen from beat to beat and reflect communication between the brain and the heart, as influenced by traffic within the sympathetic and parasympathetic nervous systems. The investigation of HRV is unique and important because it incorporates the influence of the cardiovascular and neurological systems whereas areas of interest in psychophysiology have typically focused on singular physiological systems (e.g., endocrine, cardiovascular, or reproductive systems).

HRV originally was considered to be error variance that prevented researchers from discerning valid signals of cardiac activity (Lehrer, 2007). However, knowledge of physiological processes evolved to reveal HRV's value as a variable of independent interest. The magnitude and complexity of HRV are now known to reflect important avenues for examining autonomic nervous system functioning, and low HRV has been linked to increased susceptibility to stress 
and disease (e.g., Bigger, Fleiss, Rolnitzky, \& Steinman, 1993; Carpeggiani et al., 2004; Griffin, Lake, Bissonette, Harrell, O’Shea, \& Moorman, 2005; Kim et al., 2005). Measures of HRV reflect several mechanisms working in concert to maintain a healthy level of cardiovascular as well as neural activity. When these systems are not functioning at an optimal level, an individual's stress response may be more severe. The consequence is a more deleterious effect of stress that may contribute to physical or psychological illnesses, such as cardiovascular disease, metabolic syndrome, depression, and anxiety (McGrady, 2007). It follows that obtaining and maintaining a healthy range of HRV would better equip individuals to manage stress and potentially reduce risk for or severity of numerous stress-related diseases.

In relatively recent years, investigators have drawn attention to the potential prognostic value of HRV due to relations between diminished HRV and several health problems. For example, diminished HRV has been shown to be predictive of all-cause mortality, sudden cardiac death, and other complications in patients who experienced myocardial infarction (Bigger et al., 1993; La Rovere, Bigger, Marcus, Mortara, Schwartz, 1998). Diminished HRV also has been documented in individuals with hypertension (Schroeder, Liao, Chambless, Prineas, Evans, \& Heiss, 2003), fibromyalgia (Martínez-Lavín, Hermosillo, Rosas, \& Soto, 1998), HIV and AIDS (Correia et al., 2006), and diabetic neuropathy (Boysen, Lewin, Hecker, Leichter, \& Uhlemann, 2007), among other medical conditions.

Psychological disorders or symptoms have also been investigated in relation to diminished autonomic activity. Several studies have found evidence for an association between depressive symptomology and low HRV (Agelink, Boz, Ulrich, \& Andrich, 2002; Yeragani, Roa, Smitha, Pohl, Balon, \& Srinivisan, 2002). A significant relation also was reported between anxiety and sensitivity of baroreflexes (Watkins, Blumenthal, \& Carney, 2002). Patients 
diagnosed with generalized anxiety disorder (Thayer, Friedman, \& Borkovec, 1996), panic disorder (Cohen, Benjamin, Geva, Matar, Kaplan, \& Kotler, 2000), and post-traumatic stress disorder (Cohen, Kotler, Matar, \& Kaplan, 1997; Cohen et al., 2000) also have been reported to exhibit low HRV.

Given the potential salutary effects of increasing HRV, it is important to understand how HRV may be changed. Prerequisite to that understanding, however, is knowledge of key factors involved in the measurement and physiological components of HRV. Although several methods may be used to quantify HRV (see Task Force, 1996 for a review), one of the most often used is a frequency method known as power spectral density analysis. Such analysis allows the separation of overall HRV into its distinct, composite frequency components that are each known to be associated with certain types of autonomic activity (see Figure 1). Even before the popularization of HRV as an investigatory topic, the basic contributors to each frequency component were identified. Generally, low frequency HRV was shown to be associated with

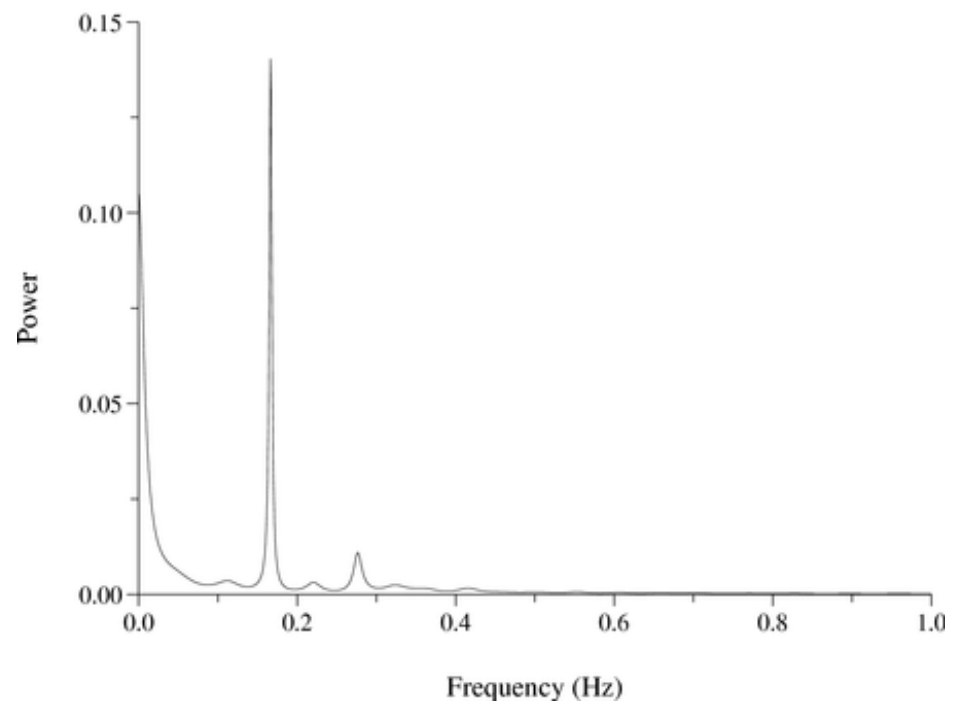

Figure 1. Power spectral density analysis graph. Waveform components between .05 and $.15 \mathrm{~Hz}$ (depicted at the far left) reflect low frequency HRV and waveform components between .15 and $.40 \mathrm{~Hz}$ reflect high frequency HRV. 
both sympathetic and parasympathetic activity, whereas high frequency HRV was linked predominantly to parasympathetic function (Akselrod, Gordon, Ubel, Shannon, Berger, \& Cohen, 1981). More details regarding each of these frequency components and their constituent physiological factors follows.

As a component of the parasympathetic nervous system, the vagus nerve innervates the heart's sinoatrial node, which is responsible for controlling heart rhythm (Berntson et al., 1997). During exhalation, the vagus nerve activates and causes heart rate (HR) to decrease, whereas inhalation causes inhibition of vagus nerve activity, disallowing vagally-mediated decreases in HR. Although there is a slight delay between respiration and change in HR during normal breathing, HR systematically varies with respiration, a phenomenon called respiratory sinus arrhythmia (RSA). Variation in HR occurs over a range of frequencies, and resting levels of RSA occur in the high frequency bandwidth of spectral waveform analysis $(0.15-0.40 \mathrm{~Hz})$ (Berntson et al., 1997; Lehrer, 2007). RSA has been suggested as a marker of parasympathetic (i.e., vagal) tone, and high frequency HRV therefore is considered to reflect vagal tone. However, because the sinoatrial node is influenced sympathetically as well, caution is merited in interpreting high frequency HRV as an unmitigated measure of vagal tone.

In addition to the high frequency range $(0.15-0.4 \mathrm{~Hz})$, there also is a low frequency bandwidth in which HRV is measured (0.05-0.15 Hz) ${ }^{2}$ (Berntson et al., 1997; Lehrer, 2007). Whereas parasympathetic tone is commonly associated with high frequency HRV, low frequency HRV is reported to reflect activity of both autonomic branches. The role of baroreceptors in HRV is typically thought to influence the low frequency range. Located in both the aortic arch and carotid sinus, baroreceptors exhibit reflex action in response to changes in blood pressure (BP) to counter sudden or extreme BP fluctuations (Berntson et al., 1997). When increases in BP 
are detected, the resulting baroreflex causes decreases in HR, which leads to decreases in BP. Conversely, baroreceptors respond to low BP by inducing increases in HR, which effectuate increases in BP. Increases or decreases in low frequency HRV are thought to be a result of (a) changes in baroreflexes that follow changes in BP, or (b) increased or decreased baroreceptor sensitivity. Baroreflexes are also thought to confer resonant properties on the cardiovascular system (Lehrer, 2007; Vaschillo, Vaschillo, \& Lehrer, 2006). Paced rhythmic breathing at a specific, individualized rate $(\approx 6$ breaths per minute, $0.1 \mathrm{~Hz})$ provides stimulation that causes $\mathrm{HR}$ and respiration to be exactly synchronized. Maximization of HRV and RSA is only achievable at this resonance frequency, which is the result of maximally efficient interactions between respiration, baroreflex activity, $\mathrm{BP}$, and $\mathrm{HR}$. This process is also considered to exercise the baroreflexes, making them generally more effective and responsive to changes in the internal and external environment.

In addition to high and low frequency HRV (i.e., frequency domain measures), HRV may also be quantified with time domain methods (Task Force, 1996). Some investigations of HRV examine both time and frequency domain outcomes (e.g., Del Pozo, Gevirtz, Scher, Guarneri, \& 2004; Hallman, Olsson, von Scheele, Melin, \& Lyskov, 2011; Karavidas et al., 2007). Although several time domain HRV outcomes exist (Task Force, 1996), many overlap in the physiological systems whose activity they reflect, so not all are discussed here. Briefly, standard deviation of normal sinus to normal sinus intervals (also called NN intervals) (i.e., SDNN) comprises all components of HRV and reflects the total power of spectral analysis. Therefore, it serves as a measure of overall HRV. However, several time domain measures are more reflective of parasympathetic activity than sympathetic activity (e.g., pNN50 and RMSSD). pNN50 is the percentage of successive NN intervals that are greater than $50 \mathrm{~ms}$, and RMSSD (in ms) is the 
square root of the mean of the squared differences of successive NN intervals. Both of these time domain parameters may be utilized, along with high frequency HRV, to measure parasympathetic influence (Task Force, 1996).

Studies have shown that compromised HRV, measured using both time and frequency based methods of assessment, pervades several undesirable physical and mental health conditions (see Wheat \& Larkin, 2010). As a marker of decreased autonomic health, restricted HRV may play a significant role in conveying risk for perseveration, if not worsening, of symptoms in a wide range of psychological and physical disorders as well as all-cause mortality. Given the potential role of reduced HRV reflecting a physiological mechanism through which psychological factors affect health, an important avenue of scientific inquiry is to identify effective treatments for increasing HRV. One promising method of intervention is using HRV biofeedback.

\subsection{Heart Rate Variability Biofeedback}

Although biofeedback has been used for many years to modify a broad range of physiological processes, it recently has been implemented as a method for changing HRV and/or baroreflexes. Biofeedback involves (a) increasing awareness and/or self-regulation of physiological activity (b) via measurement of physiological activity and provision of corresponding visual or auditory feedback (Schwartz \& Schwartz, 2003). Although the application of biofeedback to HRV is recent, biofeedback itself has a longer history. Research in the 1960s and 1970s showed that autonomic processes were not beyond voluntary control and could be conditioned instrumentally (e.g., LeFevers, 1971; McCanne \& Sandman, 1974; Schoenfeld, Matos, \& Snapper, 1967). In combination with that knowledge, the emergence of behavioral medicine, emphasis on stress and stress management research, advancement in 
physiological measurement, and other historical factors set the stage for research in various forms of biofeedback and its clinical applications. Older forms of biofeedback (e.g., HR, BP) focused on achieving a level, rather than altering the variability, of an aspect of physiology (Lehrer, 2007). Older methods of biofeedback also were less comprehensive in improving cardiovascular regulation than HRV biofeedback. For example, whereas BP biofeedback addresses improvement of BP regulation directly, likely through indirect effects on other parameters (e.g., HR regulation), HRV encompasses the interaction of several individual cardiovascular processes. Its biofeedback involves the broader mechanism of homeostasis within both cardiovascular and neural systems.

Although HRV biofeedback is a relatively new area, several studies lend support to the proposition that such treatment is effective in improving HRV. Most studies implemented a manualized HRV biofeedback protocol developed by Lehrer et al. (2000), during which the goal is to locate participants' resonant frequency and have them practice attaining and maintaining resonant frequency via breathing at the rate at which such resonance occurs $(\sim 0.1 \mathrm{~Hz}$, low frequency range). Such practice is believed to exercise the baroreflexes and improve HRV, thereby conveying beneficial health effects. Investigations of HRV biofeedback show that increases in low frequency HRV occur during biofeedback (Hassett et al., 2007; Karavidas et al., 2007; Lehrer et al., 1997; Lehrer et al. 2003; Lehrer et al., 2004; Lehrer et al., 2006).

Although most research studies share that common method of HRV biofeedback, there has been a relatively recent appearance of alternative modalities of biofeedback that are functionally similar in methods and/or objectives (e.g., Amon \& Campbell, 2008; Heilman, Handelman, Lewis, \& Porges, 2008; McCraty, Atkinson, \& Tomasino, 2003; Muench, 2008). The potential for such newer modalities to be utilized clinically for issues such as anxiety, stress 
management, or hypertension, etc., is great, especially considering their affordability. The programs also target lay persons and various health professionals as consumers and therefore are user friendly. However, there is a paucity of research dedicated to discerning whether biofeedback treatment with these instruments confers any improvement in HRV.

The emWave (formerly Freeze Framer, HeartMath Institute, Boulder, CO) is one such product that currently is marketed for use as biofeedback treatment. To this author's knowledge, no data exists indicating how many and what types (e.g., healthcare provider vs. layperson/personal consumer) of individuals or companies are utilizing emWave or related products from HeartMath. It is available to the public for purchase on the worldwide web. Whereas the protocol utilized in traditional HRV biofeedback studies emphasize breathing at the resonance frequency to produce a $\sim 0.1 \mathrm{~Hz}$ peak, treatment with the emWave focuses on training induction of positive emotional states that are associated with 'psychophysiological coherence,' as termed by its creators at the HeartMath Institute (McCraty, Atkinson, Tomasino, \& Bradley, 2006). The coherent state is characterized in the following way:

The development of heart rhythm coherence — a stable, sine-wave-like pattern in the heart rate variability waveform - is the key marker of the psychophysiological coherence mode. Heart rhythm coherence is reflected in the HRV power spectrum as a large increase in power in the low frequency (LF) band (typically around 0.1 Hz) (p. 8).

McCraty and colleagues propose that emotional training is preferable to breathing training due to the effort required to maintain paced breathing at a specified rate for an extended time period. Therefore, although the biofeedback methods differ, it is clear that emWave training embodies biofeedback session goals that functionally are similar to those implemented in historical HRV 
biofeedback research. In fact, the visual biofeedback of HRV that is provided with the emWave software is strikingly similar to that which is provided with software utilized by HRV biofeedback researchers (e.g., Lehrer et al., 2003). Further, McCraty et al. directly equate their concept of coherence with what "[Lehrer] calls "resonance"” (p. 23). Further, calculation of coherence utilizes information derived during power spectral density analysis of HRV (see Figure 2). In brief, the coherence ratio is the proportion of the waveform immediately surrounding the peak of the spectral density analysis to the remaining components of the waveform. High coherence ratios indicate that one's heart rhythm occurs consistently within the frequency band that surrounds the peak waveform, as depicted in Figure 2. In contrast, low coherence ratios are depicted by a broader display of frequencies in the spectral waveform.

\section{Heart Rhythm Coherence Ratio Calculation}

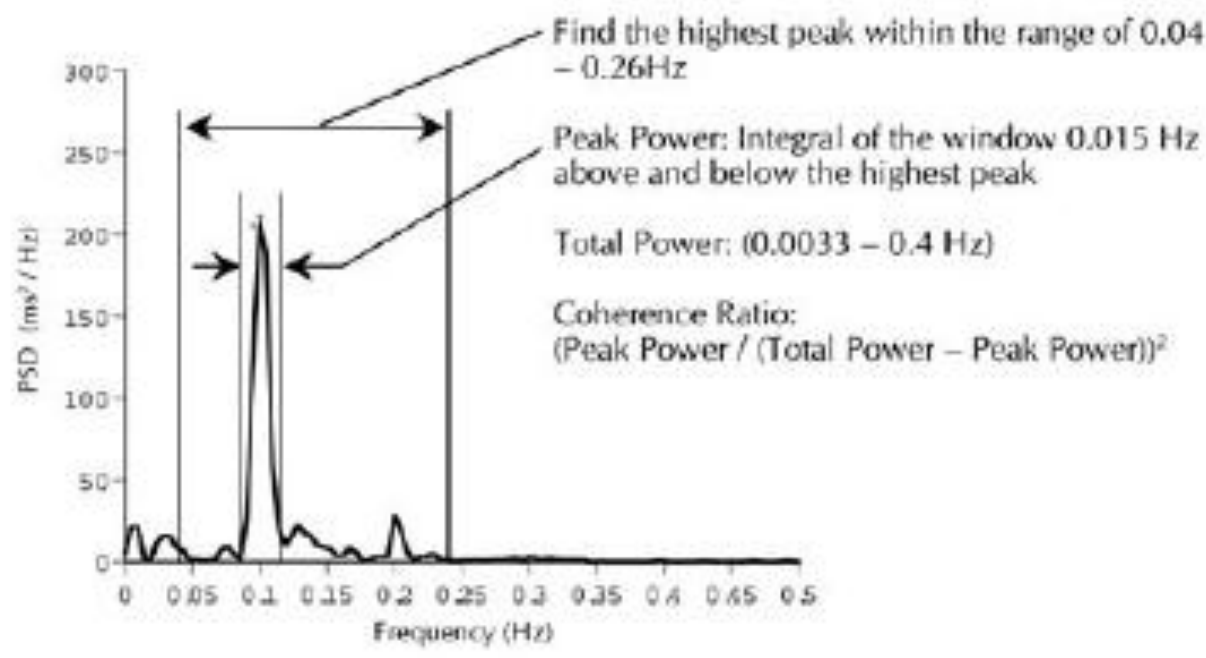

Figure 2. Utilization of power spectral density data to calculate the emWave's coherence ratio. Figure taken from McCraty et al. (2006).

It may be reasonably expected then that coherence training received through biofeedback with the emWave should produce improvement in HRV, if the training is effective. However, no 
study has been conducted specifically to validate the emWave as a tool for improving HRV via biofeedback. In fact, many interventions have implemented the emotional management techniques of the emWave or Freeze Framer programs without implementing biofeedback at all. Results of these studies indicated that treatment without the biofeedback component resulted in increased positive affect, positive attitudes, and total HRV (but not low or high frequency HRV), as well as reductions in stress, anxiety symptoms, depression symptoms, hostility, and salivary cortisol (Barrios-Choplin, McCraty, \& Cryer, 1997; Luskin, Reitz, Newell, Quinn, \& Haskell, 2002; McCraty, Barrios-Choplin, Rozman, Atkinson, \& Watkins, 1998; Rozman, Whitaker, Beckman, \& Jones, 1996; Barrios-Choplin, McCraty, Sundram, \& Atkinson, 1999). Some studies have incorporated the biofeedback component as well. Those studies showed that treatment resulted in increased workplace satisfaction, positive affect, positive attitudes, and peacefulness, as well as reductions in areas such as stress, symptoms of anxiety, symptoms of depression, social inhibition, negative affect, anger, fatigue, sleeplessness, and blood pressure (Barrios-Choplin et al., 1999; Climov, 2008; McCraty, Atkinson, Lipsenthal, \& Arguelles, 2003; McCraty et al., 2003b; McCraty, Atkinson, Tomasino, Goelitz, \& Mayrovitz, 1999; McCraty, Tomasino, Atkinson, Aasen, \& Thurik, 2000). Of the studies incorporating biofeedback, only two studies assessed HRV as an outcome. One study failed to find significant differences in HRV following treatment (McCraty et al., 2003a), whereas results of the other study indicated that middle school students receiving treatment, but not those students in a control group, exhibited increased HRV during recovery from a stressor following treatment (McCraty et al., 1999). It is difficult to make conclusions on the efficacy of the emWave program in improving HRV for several reasons. First, the results of studies that assess HRV are contradictory. Second, each of the studies suffered from serious limitations (e.g., contamination of control group, 
optional nature of biofeedback), thereby reducing confidence in their respective results. More generally, most investigations of techniques involved in the emWave program - emotion management training, biofeedback, or both — involved serious limitations that preclude confidence in the effectiveness of the program. These issues include, but are not limited to, lack of a control group, embedding of emWave components within a larger set of treatment techniques, variation within studies of treatment delivery (e.g., some participants undergo more extensive treatments or more treatment components than others).

Validation of the emWave's capacity to confer improvement in HRV, and whether those changes correlate with coherence, would lend credibility to the emWave as an affordable tool for use in improving HRV, which is a marker of physical and mental health, as outlined previously. Third-party validation is important with the emWave because the majority of research surrounding its use has been produced by the HeartMath Institute and/or its members. In fact, a portion of the research is published through the HeartMath Institute rather than peer-reviewed journals.

Another limitation of studies investigating treatment effects of the emWave, congruent with the biofeedback literature in general, is the tendency to focus on altering tonic physiological levels rather than phasic physiologic responses. Tonic levels of a given physiological parameter refer to resting levels of that parameter, whereas phasic levels refer to measures of the given parameter in response to a physical or psychological challenge. If indeed the emWave is intended to make coherence/resonance easier to achieve within the context of stress (McCraty et al., 2006), studies of its efficacy need to employ both tonic and phasic measures of HRV as outcome variables. Despite the conception that improvements in HRV may serve to buffer the effects of stress on the cardiovascular system, only one traditional HRV biofeedback study and 
one emWave study have examined whether HRV biofeedback treatment would result in improved phasic HRV responses to stress (McCraty et al., 1999; Nolan et al., 2005). In the traditional HRV biofeedback study, patients with coronary heart disease were randomized to a treatment or active control group (i.e., treatment as usual) (Nolan et al., 2005). The authors proposed that the HRV biofeedback treatment would result in increased parasympathetic recovery from stressors, as indicated by increased high frequency HRV during recovery. At posttreatment, the treatment group exhibited increases in high frequency HRV following one of three stressors and during the final recovery period following all stressors. No such significant increases were observed during any recovery period in the control group. Therefore, following the experience of vagal inhibition (i.e., decreased HRV) during stress, HRV biofeedback appeared to effectuate increases in high frequency HRV, indicating improved vagal recovery from stress.

Nolan et al.'s (2005) investigation suffered from shortcomings, however. For example, participants in the treatment group underwent HRV biofeedback after experiencing all components that the active control received (e.g., autogenic relaxation training, pleasant events scheduling). Therefore, it is possible that significant improvements in HRV recovery following stress were due to a combination of the two protocols rather than to HRV biofeedback alone. In addition, approximately $18 \%$ of the total sample was excluded from analyses due to exhibition of HRV during recovery at a frequency that was characteristic of slow, paced breathing, which they were instructed not to do. Although necessary for analytical purposes, such elimination may affect the validity of the results as those participants' may have differed from subjects whose data were analyzed regarding their cardiovascular responses to treatment or active control protocols. Potential cardiovascular differences between those who were and were not excluded 
for that reason were not analyzed. Finally, coronary heart disease patients can reasonably be expected to vary from healthy subjects in cardiovascular profiles and responses. Therefore, the study results cannot be generalized to healthy individuals.

The emWave study that implemented a stressor protocol involved middle-school student participants who were enrolled in a course in emotional competence ("Heart Smarts") (McCraty et al., 1999). The course aimed to increase the participants' ability to cope appropriately with stress during situations that were emotionally challenging, and training with the emWave was one part of the course. A subset of participants was selected to participate in a stress recovery protocol following treatment, and the treatment group was instructed to use the emWave techniques they learned during the recovery period that followed the stressor. The treatment and control groups did not differ in HRV during baseline or during the stressor. During recovery, however, the treatment group exhibited higher total and LF HRV than the control group.

Similar to the previously described study that used a stress reactivity protocol, McCraty et al.'s (1999) study exhibited significant flaws. Most importantly, biofeedback was offered to the students as part of the treatment, but was not mandatory. No analyses were performed to separate those who did and did not utilize the biofeedback component. Therefore, it is impossible to determine to what extent biofeedback was implemented and how it affected HRV outcomes. In a related vein, the techniques involved in the emWave/Freeze Framer portion of the program were included within a year-long course that taught additional skills that may have contributed to the outcome independent of the emotional management portion of treatment. Therefore, given the near absence of research investigating HRV outcomes in the context of stress reactivity protocols and the quality of the two studies that have been performed, the need to determine whether biofeedback improves reactivity to and recovery from stressors is evident. 


\section{Goals and Significance of the Proposed Study}

Due to the absence of research investigating whether biofeedback treatment with the emWave results in physiological regulation as indexed by HRV, the first goal of the proposed study was to determine whether such effects exist. Because improved HRV should serve to mitigate the effects of stress, thereby purportedly conveying decreased risk for development, worsening of, or complications following physical and psychiatric illness, the second goal of the proposed study was to determine whether reactivity to and recovery from a stressor improve following treatment with the emWave. Therefore, the following research questions were proposed:

1. Does HRV biofeedback treatment with the emWave effectuate increases in tonic HRV?

2. Does HRV biofeedback treatment with the emWave confer improvement in HRV reactivity during a stressor?

3. Does HRV biofeedback treatment with the emWave result in differences in HRV recovery after a stressor?

Answering these questions may address the following shortcomings that currently demand empirical attention in HRV biofeedback literature. Although used in full or in part in several empirical endeavors, biofeedback treatment with the emWave has not been subject to analytical scrutiny regarding its effects on HRV. No study exists to the author's knowledge that specifically aimed to validate the emWave for conveying improvement in HRV. Although several studies that utilized the emWave in some fashion have analyzed HRV outcomes, those investigations (1) were performed primarily to address questions that did not involve HRV, and (2) exhibited significant flaws that prohibit determination of whether the emWave was responsible for conferring improvements in HRV when they were present. Further, HRV 
biofeedback treatment is believed to be beneficial because it improves functioning in the cardiovascular system, ultimately resulting in partial mitigation of the stress response and its health-related consequences. Although limited attempts have been made to discern whether HRV biofeedback treatment results in improved cardiovascular responses to a stressor, the attempts were unsystematic and limited by several methodological weaknesses. The occurrence or absence of such a phenomenon, given the implication of related findings to the rationale for HRV biofeedback, deserves attention as a primary research objective. If treatment with the emWave can systematically be shown to provide improved tonic and/or phasic HRV outcomes, a valuable clinical resource that is both affordable and easily utilized may emerge for implementation in the future with a reliable evidence base.

The current project is particularly timely considering the ongoing difficulty that practitioners face in procuring reimbursement for biofeedback treatments (HRV and otherwise) (e.g., Shaffer, Neblett, \& Crawford, 2008). As the struggle for securing reimbursement continues, efforts to discern which treatments are effective and affordable are imperative. The current project was one such effort.

\section{Method}

\subsection{Study Personnel and Timeline}

Amanda Wheat, a doctoral candidate in the Department of Psychology at West Virginia University, conducted the study. Ms. Wheat's qualifications to conduct the study include a Master's degree in Psychology and three years of research involvement in the Behavioral Physiology Laboratory at West Virginia University. Data collection began during the Spring 2010 semester, and the data collection phase was completed during the Fall 2010 semester. Ms. 
Wheat assumed primary responsibility for conducting laboratory sessions described in this proposal, including overseeing one undergraduate and one graduate student research assistant.

\subsection{Participants}

Twenty-eight undergraduate and graduate student participants at West Virginia University were recruited via psychology classes using the SONA system (undergraduate students) and e-mail contact (graduate students). Participants were excluded if they smoked or used smokeless tobacco, had chronic major health problems (e.g., heart disease, cancer), or if they were on drug regimens that directly influenced heart rate and/or blood pressure (e.g., beta blockers). Participants received extra credit and/or cash for their participation, which varied based on the length of their participation (e.g., six sessions vs. eight sessions), nature of their participation (i.e., treatment vs. control group), and/or student status (undergraduate vs. graduate). Of an original pool of participants completing the online screening survey $(\mathrm{N}=325)$, 77 respondents were contacted via e-mail to participate in the lab-based portion of the study. Twenty-eight participants initiated participation in the study, and 27 (96.4\%) completed the study. A flow diagram in Figure E1 based on CONSORT 2010 recommendations (Schulz, Altman, \& Moher, 2010) provides further information regarding recruitment of the study sample.

As no HRV biofeedback studies existed at the time of study development and implementation with the same design and/or analytical strategy as the proposed study, it was difficult to estimate an effect size to determine appropriate sample size. Sample sizes in HRV biofeedback studies whose results showed short-term changes in HRV range widely, although some authors reported significant HRV results with small samples. Lehrer et al. (1997) utilized 17 participants in a three-group treatment design, whereas Hassett et al. (2007) and Karavidas et al. (2007) implemented open-label treatment studies without control groups with 12 and 11 
participants, respectively. Each of those studies found significant improvement in one or more HRV outcomes. It should be recognized that those changes were not evident from pre-treatment to post-treatment, which is common across such traditional HRV biofeedback studies. However, because significant HRV findings were present, it is unlikely that the analyses were underpowered. It seems more likely that the treatment protocol implemented in those studies was not configured as it stood to support long-term pre-treatment to post-treatment improvements. Treatment with the emWave may better confer such outcomes. Therefore, the current study's aim was inclusion of twenty-four participants, and 28 participants enrolled. Although analyses including as few as 11-17 participants resulted in significant findings, collecting data for a few more participants appeared reasonable to increase likelihood of sufficient power for detecting treatment effects.

\subsection{Experimental and Analytical Design}

Recruited participants were randomly assigned to either the treatment or control group. At pre-treatment, both groups participated in a stress reactivity protocol during the initial lab visit. The treatment group then received a PC-based biofeedback treatment across several lab visits. All participants then underwent a post-treatment assessment identical to the pre-treatment assessment. A mixed factors Group (Treatment, Control) by Session (Pre-Treatment, PostTreatment) research design was employed, with Group serving as between subjects factor and Session as the repeated measures factor.

\subsection{Measures}

3.4.1. Demographics. Participants completed a demographics questionnaire including items related to participant characteristics (e.g., age, sex), health behaviors (e.g., exercise, 
smoking), health problems (e.g., chronic illness), and medications known to have cardiovascular effects that could confound HRV data (see Appendix A).

3.4.2. Heart Rate Variability. HRV was measured using HR data gathered with a Polar (Lake Success, New York) RS800CX heart rate monitor. Data obtained from the Polar monitor have been shown to correlate highly and significantly with ECG-derived measures of HR during rest periods and during performance of stressful tasks (Goodie, Larkin, \& Schauss, 2000). This device functions by transmitting ECG signals from a sensor strapped around the participants' chest to a wristwatch in an adjoined room. Via a USB device, the wristwatch was wirelessly connected to a computer where data collection was monitored real-time by the experimenter.

3.4.3. Self-report Measures. Self-report measures of psychological symptoms and distress were included for purposes of comparing results relevant to these outcomes with previous studies implementing emWave training to some extent that used the same (or similar) measures. Additionally, state affect was measured to supplement measures of physiological indices of reactivity and recovery.

The Brief Symptom Inventory (BSI; Derogatis, 1993) provided a global measure of distress (i.e., Global Severity Index) and nine specific subscale scores (i.e., Somatization, Obsessive-compulsive, Interpersonal Sensitivity, Depression, Anxiety, Hostility, Phobic Anxiety, Paranoid Ideation, Psychoticism). Participants responded to 53 items on a 5-point Likert-type scale to indicate intensity/distress related to various symptoms. The BSI exhibits adequate internal consistency, test-retest reliability, and convergent and construct validity (Derogatis \& Melisaratos, 1983). Further, correlations with the Symptom Checklist-90, the parent form of the BSI, were shown to be high. Following are Cronbach's alpha values for the BSI subscales in the current study: Somatization (pre-test: 0.73; post-test: 0.79), Obsessive- 
compulsive (0.86; 0.90), Interpersonal Sensitivity (0.76; 0.83), Depression (0.82; 0.86), Anxiety (0.76; 0.84), Hostility (0.73; 0.68), Phobic Anxiety (0.64; 0.90), Paranoid Ideation (0.72; 0.80), and Psychoticism (0.50; 0.62). On average, Cronbach's alpha for pre-test subscales was 0.72 , and for post-test subscales was 0.80. Cronbach's alpha for the Global Severity Index at the pretest session was 0.95 , and was 0.96 at the post-test session.

The Perceived Stress Scale (PSS) indexed stress with 14 items rated on a Likert-type scale (Cohen, Kamarck, \& Mermelstein, 1983). Participants indicated how often they had certain experiences in the previous month (e.g., "How often have you felt that you were on top of things?"). Sufficient alpha coefficient reliabilities (i.e., $\approx 0.85$ ) have been demonstrated with the PSS, and PSS scores also were shown to correlate significantly with life event and depressive symptom scores. Cronbach's alphas for the current study for the scale at pre-treatment and posttreatment were 0.88 and 0.89 , respectively.

Finally, the Multiple Adjective Affect Checklist - Revised (MAACL-R; Zuckerman \& Lubin, 1985) measured state affect during the stress reactivity protocol. The MAACL-R contains five subscales: Anxiety, Depression, Hostility, Positive Affect, and Sensation Seeking. Participants indicated which of 66 adjectives described how they felt at baseline and during each stressor. Validity and reliability have shown to be satisfactory for an earlier version of the instrument, and subscales of the currently proposed and earlier version were high (Lubin, Van Whitlock, Reddy, \& Petren, 2001). Further, all subscales have been shown to have high internal consistency, with an averaging .88 across subscales. As the MAACL-R was administered three times (i.e., rest, reactivity, recovery) at the two assessment sessions (i.e., pre-test and post-test), internal consistency was computed 6 times for each subscale for the current study. KuderRichardson values were: Anxiety (range $=0.22-0.81$; average $=0.49)$, Depression $(0.36-0.78$; 
0.58), Hostility (0.46-0.75; 0.67), Positive Affect (0.50-0.86; 0.69), and Sensation Seeking (0.14$0.68 ; 0.47)$. It should be noted that low internal consistency values are likely reflective of the small sample and low variance due to many or all participants endorsing few items, or sometimes none, at some points of measurement.

\subsection{Procedure}

Following their provision of informed consent, participants completed a demographic measure online to obtain standard information (e.g., age, gender) and to garner sufficient data about health issues so exclusion of ineligible participants occurred prior to inviting qualified participants to the lab. Participants were randomly assigned to treatment or control groups using a list of odd or even numbers from a random number table. After randomization, participants attended a lab session during which the pre-treatment stress reactivity assessment occurred, which is described below. Following the pre-treatment assessment, participants completed activities corresponding to their group assignment. When such activities were completed, as outlined below, a post-treatment assessment occurred that mirrored the pre-treatment assessment.

3.5.1. Stress Reactivity Protocol. During the first lab visit, participants completed consent and HIPAA authorization forms, at which time their participatory role in the study was explained. The experimenter collected height and weight data in addition to assessing for alcohol, caffeine, and nicotine use within the past two hours via self-report. Engagement in vigorous exercise was assessed at that time also. Participants denied engaging in such activities within two hours of session attendance, so none were excluded from the study or rescheduled for later participation. Before physiological assessment, participants completed self-report measures. Participants then equipped themselves with the Polar monitor in the absence of the experimenter, and the experimenter tested accurate placement of the Polar by examining data transmission to 
the computer. Participants were instructed to remain seated in a chair and to remain relatively still with feet flat on the floor while HR data were collected during a 15-minute rest period. Data from the final 5 minutes of the rest period were used in analyses. Following completion of the rest period, but before the first stressor, participants completed the first MAACL-R.

Participants then underwent a stress reactivity protocol. Specifically, a mental arithmetic task required that the participant count aloud and backward from 9,000 in increments of seven. Participants were encouraged to continue from the last place they remembered if they lost track of counting during the task. The task was five minutes in length. Following the mental arithmetic task, there was a five-minute recovery period during which they completed the second MAACLR. After the recovery period, participants engaged in an isometric hand-grip task (Lafayette Instruments hand dynamometer model 78010, Lafayette, USA). The investigator assessed participants' maximum grip strength, and the participants were instructed to maintain $30 \%$ of that grip strength for 3 minutes. Again, another five-minute recovery period followed, and participants completed the third and final MAACL-R at that time.

3.5.2. Treatment Protocol. The emWave PC Stress Relief System (“emWave," HeartMath, LLC, Boulder, Colorado) provided HRV biofeedback treatment. The emWave includes a fingertip plethysmograph that transfers heart rate data to a software program through which biofeedback is provided. Data from the sensor is transformed to display participants' heart rhythm in real time. In addition, graphs depicting the HRV power spectrum or pulse wave were displayed. The third biofeedback component involves coherence. There are three bars of varying colors, and each responds to low, medium, or high coherence levels. This visual biofeedback provided information to the participant to assist in physiological and emotional self-regulation while they implemented the quick coherence technique taught with emWave (see below). 
Following their initial assessment, participants in the treatment group worked with the experimenter to customize a schedule for biofeedback training. HRV, HRV biofeedback, and how HRV is related to health were explained at the first biofeedback visit. In addition, the emWave's "quick coherence technique" was reviewed and practiced during the first treatment visit. The technique involves attending to the physical area of the heart ("heart focus"), breathing deeply ("heart breathing"), and producing positive emotion ("heart feeling"). A more detailed description of these steps is listed in Appendix B. All treatment participants wore the Polar heart rate monitor during each entire biofeedback training session to allow for assessment of physiological change across training. Because individuals progressed in exhibiting adequate coherence levels at different paces, the length of training time was variable concerning total sessions completed. Guidelines provided by HeartMath in their Practitioner's Guide (Culbert, Martin, \& McCraty, 2007) suggest that four to six sessions are typically recommended, so a minimum for four completed sessions prior to completion of the post-test assessment was imposed. Sessions were 32 minutes in length, and they occurred approximately weekly. Days between the initial and follow-up visits ranged from 28 to 84 days. Control participants were yoked to treatment participants to assure equivalence of length of time between visits.

Participants practiced attaining increasing levels of moderate and/or high coherence as treatment progressed, thereby reducing the level of low coherence during sessions. Participants remained in treatment until they achieved $<50 \%$ low coherence (as suggested in the emWave practitioner's guide) and evidenced a distinguishable peak within the low frequency range on the power spectrum within a training session. The latter criterion is not typically a part of emWave treatment. However, it was incorporated into the study protocol to assure that more typical HRV measures included in traditional HRV biofeedback were attended to by participants. Further, that 
criterion should naturally be reached if coherence indeed is reflective of a peak around $0.1 \mathrm{~Hz}$ in the power spectrum, as noted earlier. Further, the above listed criteria had to be met first at the emWave's low level of difficulty, and then again at the medium level of difficulty. If participants met those criteria within four sessions, they then completed the post-test session. For participants who did not meet these criteria by conclusion of the fourth session, they continued for up to four additional sessions before completing the post-test.

Throughout treatment, participants were instructed to practice the techniques learned with the emWave program. They were instructed to do so for 10 minutes each day at a time when they were relatively calm and in an environment with as little distraction as possible. They also were instructed to practice the techniques when perceiving stress throughout the day. Participants were provided logs (see Appendix C) to record when they utilized the techniques and whether it occurred during a time of calm or of stress. The experimenter reviewed the logs with participants at each visit prior to beginning biofeedback so any questions or concerns the participants had could be addressed.

3.5.3. Post-treatment Assessment. Following completion of the treatment sessions, the experimenter scheduled the final post-test session with participants. Visits for control group participants were yoked as closely as possible to visits for participants in the treatment group. Each time a treatment participant was scheduled for their post-test assessment, the experimenter scheduled a post-test assessment with the control participant who completed his or her initial assessment at the time closest to the corresponding treatment participant. The stressor protocol administered during the initial assessment was repeated. Using the quick coherence technique alters physiology and would obscure the physiological response normally expected during stress reactivity and recovery. Therefore, treatment participants were instructed not to use the 
techniques learned during their biofeedback sessions. The writer monitored the heart rate waveform on the computer real-time during the assessment as well, as a distinct waveform is detectable when participants use the quick coherence technique, to detect whether participants were adhering to these instructions. Participants also were debriefed during the final session, and those in the control group were offered the opportunity to undergo biofeedback treatment.

\section{Results}

\subsection{Data Reduction and Preparation for Analyses}

4.1.1. Self-report Outcomes. Missing data for self-report outcome measures were extremely scarce in the current sample. Three participants omitted responses for between one and three items on the BSI. Following guidelines in the published BSI manual (Derogatis, 2001), calculation of scale scores was adjusted downward based on number of items with responses rather than absolute number of scale items. According to the manual, integrity is maintained with up to $25 \%$ of items missing. The largest proportion missing in the current sample among the three participants with missing data was $5.7 \%$. Additionally, one participant failed to respond to one item on the PSS at the pre-test assessment. Mean imputation was utilized in this case. Otherwise, data were intact for BSI, PSS, and MAACL-R measures.

Skew and kurtosis were examined for BSI, PSS, and MAACL-R data. No abnormalities were present for PSS scores. Skew and/or kurtosis were significant (i.e., exceeding absolute value greater than 3 or 10, respectively) for the following scales at the pre- and/or post-test assessments: MAACL-R Positive Affect, MAACL-R Hostility, MAACL-R Sensation Seeking, BSI Anxiety, and BSI Phobic Anxiety. Therefore, data for both sessions and all task periods for these subscales were logarithmically transformed to promote normality of data. Following the transformation, skew and kurtosis were within acceptable limits with the exception of (1) the 
Hostility subscale of the MAACL-R during the rest period at the pre-test session ( $\mathrm{skew}=4.32$; kurtosis $=19.31)$ and $(2)$ the Phobic Anxiety subscale of the BSI at the post-test session (skew = 3.52 , kurtosis $=14.06)$. Inspection of the data revealed that the elevation was due to two participants who endorsed at least one item on each scale; the remaining respondents endorsed no items on either scale. However, there was no indication of error in data entry or subject report on the measure. Rather, the data point seemed to genuinely reflect higher levels of self-reported hostile affect and phobic anxiety among outliers. To maintain the integrity of the data, the outliers were not removed.

4.1.2. Physiological Outcomes. Heart rate data for each period of pre- and post-treatment assessment sessions (i.e., Initial Rest Period; Mental Arithmetic Task; Mental Arithmetic Recovery; Handgrip Task; Handgrip Recovery) were collected using the Polar monitor and imported to Kubios HRV Analysis Software v2.0 (Biosignal Analysis and Medical Imaging Group, Kuopio, Finland) for preparation and analysis of HRV. Within the Kubios program, R-R interval data were visually inspected for aberrant or missing data. Low-level artifact correction was employed in these cases using the artifact correction feature of Kubios, which applies an interpolation method to correct erroneous data. In six instances, heart rate data were not transmitted effectively from the Polar monitor during one or two portions the stressor protocol, resulting in a gap in R-R interval data (e.g., 15 seconds during baseline). These sections of data were removed due to clear invalidity, and analyses were performed both including and excluding these participants' data. Findings did not differ meaningfully between analytical approaches, so data reported below include these participant's data.

Following screening and error correction of the physiological data, LF and HF HRV values were logarithmically transformed. As frequency domain HRV data (i.e., LF HRV and HF 
HRV) are known to suffer from skewness, implementing log transformation is a widely encouraged and implemented practice (e.g., Kuo et al., 1999; Pinna et al., 2007).

\subsection{Preliminary Analyses}

Several analyses were deemed necessary prior to conducting primary study analyses, including: (a) examining demographic characteristics of the treatment and control groups to assure group equivalence on pertinent variables; (b) examining relations between HRV and potential covariates; (c) demonstrating that the tasks chosen to elicit stress responses evoked changes in measures of cardiovascular functioning; (d) determining whether the two stressor tasks chosen for this study elicited comparable cardiovascular and affective responses; (e) demonstrating that participants in the treatment group evidenced changes in coherence using the emWave biofeedback protocol; and (f) examining whether participants in the treatment group adhered to homework practice using the techniques taught in between training sessions.

4.2.1. Group Differences on Demographic Variables. Analyses were performed (i.e., $t$, $\chi^{2}$, or $\left.\varphi\right)$ to determine whether treatment and control groups differed regarding the demographic characteristics of sex, age, height, weight, BMI, race, ethnicity, parent income, and student status (i.e., graduate versus undergraduate). As depicted in Table 1, no significant differences were evident on any demographic characteristic (all $p s>.05$ ).

4.2.2. Potential Covariates. As sex, age, and BMI are often associated with heart rate and/or HRV (e.g., Bonnemeier et al., 2003; Felber Deitrich et al., 2006), correlational analyses were performed to examine the presence of significant relations between these three variables and HRV data to identify potential covariates for use in the primary study hypotheses. These analyses were conducted using Pearson's correlation coefficients. As depicted in Table 2, age 
was related to several measures of $\mathrm{HRV}$ and was therefore incorporated as a covariate in all main analyses involving physiological data.

4.2.3. Evidence of Stress Reactivity to Tasks. To validate the two tasks chosen to assess participants' stress responses in this study, heart rate level during each task was examined to confirm that heart rate increased significantly from the pre-task rest period to the task period. During mental arithmetic and handgrip tasks at both the pre- and post-test sessions, heart rate was significantly higher in comparison to the pre-task rest period from the same session (all $p$ s < .001 ; see Table 3).

4.2.4. Task Differences. To determine whether significant differences existed in reactivity to the two stressor tasks, 2 (Group) X 2 (Task) ANCOVAs were performed using pretreatment HRV, covarying respective pre-task resting values. As depicted in Table 4, no Group $\mathrm{X}$ Task interactions were significant for any HRV measure (all $p \mathrm{~s}>.05$ ), nor were any main effects for Task significant (all $p s>.05$ ). Comparable analyses were conducted on HRV reactivity values at post-treatment with comparable results (see Table 4). Based upon these findings, HRV data were averaged across the two tasks at both pre- and post-treatment phases for conducting the primary study analyses.

Analysis of task differences in affective responding revealed that while no significant task differences were noted for Anxiety, Depression, Hostility, and Positive Affect, there was a significant difference in Sensation Seeking between the math and handgrip tasks. Therefore, data for Sensation Seeking was analyzed separately for the two stressor tasks. Data for the remaining MAACL-R subscales (i.e., Anxiety, Depression, Hostility, Positive Affect) were aggregated across tasks. 


\subsubsection{Heart Rate Variability and emWave performance during treatment sessions.}

Participants completed a minimum of four sessions, and a maximum of eight sessions. Sessions occurred approximately weekly.

Prior to examining primary study hypotheses, it is important to demonstrate that participants in the treatment group actually acquired some skill at regulating their physiologies during training sessions. Two types of data from treatment group participants were examined for this purpose: HRV data collected during biofeedback training sessions from the Polar monitor, and values from each training session based upon coherence information from the emWave program. The following point system was developed to determine how successful study participants were in completing emWave coherence training for each session:

\begin{tabular}{|c|l|c|}
\hline Point Type & Criteria & Point Value \\
\hline \multirow{4}{*}{ Session Difficulty } & Low Difficulty & 1 \\
\cline { 2 - 3 } & Medium Difficulty & 2 \\
\cline { 2 - 3 } & High Difficulty & 4 \\
\cline { 2 - 3 } & Very High Difficulty & 1 \\
\hline \multirow{5}{*}{ Coherence } & $\begin{array}{l}<50 \text { (of 100) coherence points in the low } \\
\text { coherence category for each entire session }\end{array}$ & \multicolumn{2}{|c|}{0} \\
\cline { 2 - 3 } & $\begin{array}{l}>50 \% \text { (of 100) coherence points in the low } \\
\text { coherence category for each entire session }\end{array}$ & 1 \\
\hline \multirow{2}{*}{ HRV Points } & Distinct peak was apparent in the LF range & 0 \\
\cline { 2 - 3 } & No distinct peak was apparent in the LF range & \multicolumn{2}{|c|}{1} \\
\hline
\end{tabular}

Using this schema, the maximum point value earned for a single training session was six; four session difficulty points for engaging in training with very high difficulty, one coherence point for exhibiting more than $50 \%$ of data points in the high coherence range, and one HRV point for displaying a distinct peak in the LF frequency range. All participants completed four sessions, but some completed up to eight sessions because they did not meet the established 
training criteria (i.e., $<50 \%$ data points in the low coherence range and a distinct peak in the LF range for both low and medium levels of difficulty) by the end of the fourth training session. For participants completing fewer than eight sessions, the final observed data point from the last completed session (e.g., Sessions 4 or 6) was carried forward for each participant through session 8.

HRV values obtained from the Polar HR Monitor during training and point accumulation from the emWave protocol for treatment group participants were compared across training trials using repeated measures ANCOVAs. No changes in HRV across treatment sessions were apparent when analyzed in a variety of ways (e.g., examining changes across the first four sessions, between the first and last session, between the beginning and end of each or all sessions) $(p s>.05)$. Table 5 contains relevant data for the comparison of HRV between the first and the final treatment session, illustrating the lack of change in HRV from the initiation to conclusion of treatment. Conversely, an increase in emWave performance points was apparent across sessions, $F(1,10)=61.73, p<.001$, wherein performance improved over time on average across participants (see Figure E2, Table 6). Post-hoc analyses revealed that sessions 1-3 differed from all other sessions $(p s<.01)$, whereas sessions 4-8 did not differ significantly from each other $(p s>.05)$, likely because data for participants completing fewer than 8 sessions were carried forward for these analyses.

4.2.6. Home Practice of Treatment. Thirteen of 14 treatment participants completed homework logs in between treatment sessions. One participant did not submit logs, but reported practicing the technique. For the remaining 13 participants, practice of the quick coherence technique reportedly occurred during both calm and stressful times during their daily lives. The average number of minutes practiced during times of calm and of stress per week was 44.29 (SD 
$=24.48$; range: 9-89) and $47.37(\mathrm{SD}=37.13$; range: 7-110) minutes, respectively. Overall, the average amount of homework practice was $98.46(\mathrm{SD}=60.79$ range: $17-197)$ minutes per week. The average number of emWave performance points earned during each session and the average number of minutes practiced per week were not significantly correlated $(r=.19, p>$ $.05)$.

\subsection{Primary Analyses}

Mixed factors 2 (Group: Treatment, Control) x 2 (Session: Pre- vs. Post-treatment) analyses of covariance (ANCOVAs) were performed to test for differences in tonic HRV, phasic HRV reactivity to stress, and HRV recovery from stress before and after treatment. As mentioned above, age was used as a covariate for these analyses. Dependent variables included LF and HF HRV, as well as RMSSD, pNN50, and SDNN. To assess for differences in selfreported affect from pre- to post-treatment, comparable 2 × 2 ANCOVAs were conducted, with BSI, PSS, and MAACL-R data serving as dependent variables. For analyses of reactivity, pretask rest period parameters were also included as covariates to control for potential differences in resting levels of the observed parameters.

\subsubsection{Physiological Outcomes}

4.3.1.1. Tonic Measures of Heart Rate Variability. Results of the ANCOVAs on tonic measures of HRV measured from the initial rest period of the pre- and post-treatment assessment sessions revealed neither significant main effects for Session nor Group by Session interactions (see Table 7). This indicates that treatment using the emWave protocol did not influence tonic HRV in study participants. However, there were significant main effects for Group for both LF HRV and SDNN. At rest, the treatment group exhibited higher LF HRV $\left(E M M=7.20_{\log } \mathrm{ms}^{2}, S E\right.$ $=0.21)$ and $\operatorname{SDNN}\left(E M M=69.59 \log ^{2} \mathrm{~ms}^{2}, S E=4.16\right)$ than the control group $(\mathrm{LF} \mathrm{HRV}, E M M=$ 
$\left.6.40{ }_{\log } \mathrm{ms}^{2}, S E=0.22 ; \mathrm{SDNN}, E M M=51.27{ }_{\log } \mathrm{ms}^{2}, S E=4.31\right)$. Despite randomized group assignment, participants in the treatment group evidenced higher levels of autonomic stability than control participants, a phenomenon that was evident both prior to and following the treatment phases. Based upon these findings, resting measures of HRV were incorporated as covariates in all subsequent analyses of HRV parameters. As such, results of subsequent analyses of task reactivity to stress and recovery from stress were not influenced by any group differences in resting physiology.

4.3.1.2. Phasic Heart Rate Variability Reactivity to Stress. Using aggregated reactivity data across both tasks (i.e., mental arithmetic and handgrip), Group X Session ANCOVAs were performed on LF HRV, HF HRV, SDNN, RMSSD, and pNN50 during the stressor tasks, covarying age and corresponding resting levels of HRV. A significant Group X Session interaction was evident for pNN50. The treatment and control groups did not differ regarding HRV reactivity to stress during the pre-treatment session, $F(1,23)=0.60, p>.05$, but showed significant group differences in pNN50 stress reactivity values at the post-treatment session, $F$ $(1,23)=6.25, p<.05, \eta_{\mathrm{p}}^{2}=0.21$ (See Table 8 for ANCOVA Summary Table and Figure E3). Specifically, the treatment group exhibited higher pNN50 stress reactivity $(E M M=25.58$ percent, $S E=2.38)$ than the control group $(E M M=16.98$ percent, $S E=2.47)$ at the post-test session. Adopting the .05 level of confidence, no significant main effects or interactions were observed for LF HRV, HF HRV, SDNN, or RMSSD reactivity (all $p$ s > .05; see Table 8).

4.3.1.3. Heart Rate Variability Recovery from Stress. Analyses on all measures of HRV recovery following exposure to stressor tasks yielded no main effects or interactions for LF HRV, HF HRV, SDNN, RMSSD, and pNN50 recovery ( $p$ s > .05; see Table 10). 
4.3.1.4. Exploratory analyses of Treatment Responders. Two participants progressed less consistently and expeditiously than the other 12 participants in the treatment group, raising the possibility that their gains may have been different in quality or quantity than the remaining participants who engaged with the emWave treatment. The main analyses explained above were repeated excluding these two participants to explore whether their inclusion influenced study findings.

The interaction between Group and Session for pNN50 reactivity remained significant, and comparable interactions reached statistical significance in the exploratory analyses for LF HRV, SDNN, and RMSSD (see Table 9). Follow-up analyses evidenced that exclusively in the treatment group, $\operatorname{LF}\left(\eta_{\mathrm{p}}{ }^{2}=0.58\right), \operatorname{SDNN}\left(\eta_{\mathrm{p}}{ }^{2}=0.50\right), \mathrm{pNN} 50\left(\eta_{\mathrm{p}}{ }^{2}=0.78\right)$, and RMSSD $\left(\eta_{\mathrm{p}}{ }^{2}=\right.$ 0.61) were higher during stress during the post-treatment session relative to the pre-treatment session $(p s<.05)$. The control group showed no significant changes in LF HRV, SDNN, pNN50 or RMSSD in response to stress from pre- to post-treatment sessions (all $p \mathrm{~s}>.05$ ). The interaction for HF HRV was not significant. Finally, consistent with the main analyses including all study participants, there were no apparent treatment effects on HRV recovery following stress when conducting these analyses using only treatment responders.

\subsubsection{Supplementary Self-report Measures}

4.3.2.1. MAACL-R. As shown in Table 11, no significant Group X Session interactions were observed for measures of affective response to stress using the MAACL-R. This indicates that the emWave training did not influence affective response to the laboratory stressors used in this study. Several main effects for Session were detected, including Sensation Seeking during the mental arithmetic task, $F(1,24)=6.44, p<.05, \eta_{\mathrm{p}}{ }^{2}=0,21$, and Depression, $F(1,24)=7.82, p$ $=.01$, and Anxiety, $\eta_{\mathrm{p}}{ }^{2}=0.24, F(1,24)=4.61, p<.05, \eta_{\mathrm{p}}{ }^{2}=0.16$, during pre-task rest periods. 
During the mental arithmetic task, all participants reported higher sensation seeking at the posttreatment session $(M=0.63, S D=0.79)$ compared to the pre-treatment $\operatorname{session}(M=0.41, S D=$ 0.84). Self-reported depressed and anxious affect decreased on average from pre-treatment (Depression: $M=0.78, S D=1.19$; Anxiety: $M=1.37, S D=2.08$ ) to the post-treatment assessment sessions (Depression: $M=0.37, S D=0.84$; Anxiety: $M=0.56, S D=0.85$ ). No main effects of Group were observed for any measure of affective responding.

4.3.2.2. Perceived Stress Scale. A 2 (Group) x 2 (Session) ANOVA on PSS scores revealed a significant main effect for Session, $F(1,24)=6.23, p<.05, d=0.45$ (see Table 11). Self-reported stress decreased from pre- $(M=38.87, S D=7.87)$ to post-treatment $(M=35.37$, $S D=7.52$ ) assessment sessions for all study participants. Decreases in self-reported stress levels did not differ between treatment and control groups, however.

4.3.2.3. Brief Symptom Inventory. All nine subscales of the BSI, as well as the Global Severity index, were analyzed using 2 (Group) x 2 (Session) ANOVAs (see Table 11). Main effects for Session were observed for the Global Severity Index, $F(1,25)=5.51, p<.05, \eta_{\mathrm{p}}{ }^{2}=$ 0.18 , and the Anxiety, $F(1,25)=5.44, p<.05, \eta_{\mathrm{p}}{ }^{2}=0.17$, and Somatization, $F(1,25)=5.24, p<$ $.05, \eta_{\mathrm{p}}{ }^{2}=0.17$, subscales; in all cases, reports of symptom-related distress decreased significantly between the pre- and post-treatment assessment sessions. A main effect for Group emerged for the Anxiety subscale as well, $F(1,25)=4.28, p<.05, \eta_{\mathrm{p}}{ }^{2}=0.15$, wherein the treatment group reported higher levels of anxiety than the control group. No other main effects or interactions were significant for any remaining BSI measures.

\section{Discussion}

The current study was designed to empirically address gaps in the HRV biofeedback literature, specifically pertaining to the delivery of HRV biofeedback using the emWave system. 
Although it may be anticipated that improvement in HRV would be secondary to improvement in coherence through biofeedback training with the emWave, no studies to date have been designed expressly to examine this possibility. Prior investigation of the efficacy of biofeedback training via emWave exhibited conflicting findings regarding physiological effects and contained significant flaws in method, making it difficult to draw any firm conclusions. Therefore, one main aim of this study was to determine whether emWave treatment resulted in changes in tonic HRV. Another limitation of emWave, and the larger HRV, biofeedback literature is that little interest in examining the effects of training on phasic physiological responses to stress is evident. As HRV biofeedback may exert clinical improvements through improving the physiological response to stress, examining treatment effects on change in phasic physiological responses to stress is critical. Therefore, the other aims of the current study were to test whether emWave treatment conferred improvement in HRV reactivity during a stressor and in HRV recovery following stress.

\subsection{Effects of emWave Training on Physiological Measures}

Linear, additive changes in HRV indices were not apparent across biofeedback training sessions in this study. Although acute increases in LF HRV during individual biofeedback sessions are apparent in traditional HRV biofeedback studies, evidence also suggests that cumulative and significant improvements in HRV across treatment visits in biofeedback sessions do not occur (Wheat \& Larkin, 2010). In a recent study, coherence values based on participants' performance during emWave biofeedback sessions were calculated to examine whether coherence changed across treatment sessions (Henriques, Keffer, Abrahamson, \& Horst, 2011). According to the investigators, increases on coherence were expected to accompany the documented decreases in reported anxiety across time. Counter to the authors' expectations, 
however, improvement in coherence across 4 weeks of biofeedback sessions did not occur. This finding is consistent with the traditional HRV biofeedback literature in which clinical effects are frequently observed despite consistent and/or significant changes in underlying physiology. For example, Karavidas and coauthors (2007) utilized the traditional HRV biofeedback protocol with a depressed sample and found that improvements in depression at the fourth biofeedback session appeared to be coupled with improvements in HRV. However, improvements in HRV did not persist across future sessions, whereas improvements in depressive symptoms persisted.

In order to examine changes in physiology occurring during emWave training, a scoring rubric was developed for use in the current study to assess change and to dictate progression of each participant through treatment. Despite lack of observable change in traditional HRV measures (e.g., LF HRV, HF HRV, SDNN) during training, improvement in emWave coherence scores and LF power spectrum peaks were detected across biofeedback treatment sessions. This suggests that participants acquired skill over time in manipulating coherence as presented by the emWave training protocol, but that these changes were not paralleled by changes in HRV.

5.1.1. Tonic Heart Rate Variability. An effect of emWave treatment on tonic, or resting, levels of HRV was not apparent in any measure of HRV in our study. Participants receiving emWave treatment did not evidence significant change in resting LF, HF, SDNN, pNN50, or RMSSD measures of HRV from the pre-treatment to post-treatment periods. One previous emWave study examined changes in physiology following treatment and found that individuals receiving emWave treatment showed increases in the ratio of LF HRV to HF HRV (McCraty et al., 2003a), a somewhat controversial measure of sympathovagal balance (Berntson et al., 1997). However, this change was not contrasted against a control group so it is unclear whether this effect was due to treatment or some unrelated factor (e.g., time) (McCraty et al., 
2003a). Also, no changes were noted in resting LF HRV, HF HRV, SDNN, or RMSSD in their study. Therefore, the current results largely replicate the finding that emWave treatment does not appear to change resting levels of HRV. The current study was designed explicitly to examine changes in resting HRV following emWave treatment. Therefore, no compelling evidence exists to date to support that tonic HRV is altered via emWave treatment.

The current results also are largely consistent with the traditional HRV biofeedback literature. Two traditional HRV biofeedback studies showed evidence of change in tonic SDNN or total HRV following HRV biofeedback treatment (Del Pozo et al., 2004; Zucker, Samuelson, Meunch, Greenberg, \& Gevirtz, 2009), but suffered several methodological shortfalls.

Conversely, several other studies with greater methodological rigor failed to document changes in tonic SDNN or total HRV (Karavidas et al., 2007; Lehrer et al., 2003; Lehrer et al., 2004; Swanson et al., 2009). One recent investigation of traditional HRV biofeedback documented changes in resting LF HRV (Hallman et al., 2011). However, this finding is not commonly observed, and the majority of studies have failed to find evidence for changes in resting or tonic LF HRV (Hassett et al., 2007; Karavidas et al., Lehrer et al., 2003; Lehrer et al., 2004). Differences in tonic HF HRV have not been found in any study using HRV biofeedback (Cowan et al., 2001; Hassett et al., 2007; Hallman et al., 2011; Karavidas et al., 2007; Lehrer et al., 2003), and differences in tonic RMSSD and pNN50 have not been examined prior to the current investigation. Therefore, absence of change in tonic measures of HRV following training with emWave biofeedback in this study is congruent with the majority of studies using traditional HRV biofeedback and the limited research using the emWave protocol. Available evidence suggests that HRV biofeedback does not reliably confer changes in resting HRV. 
Absence of changes in tonic measures of HRV may be considered evidence against the effectiveness of HRV biofeedback in general and in emWave treatment specifically, but caution must be exercised in this interpretation. Although changes in tonic HRV would certainly be beneficial and lend credence to the efficacy of HRV biofeedback, such changes may not be needed in order to confer therapeutic clinical advantages. For example, change in HRV (phasic HRV) in response to internal or environmental stressors may represent a more important outcome associated with biofeedback interventions than any potential change in tonic level of HRV. Therefore, improved phasic HRV in the context of stress may be more relevant and compelling than changes in tonic HRV.

5.1.2. Phasic Heart Rate Variability. Evidence was limited in the current study for changes in phasic HRV following emWave treatment. Neither the control nor treatment group showed evidence of change in LF HRV, HF HRV, SDNN, or RMSSD during stress. However, the treatment group exhibited higher pNN50 during stress relative to the control group at the post-treatment session. As pNN50 is largely indicative of parasympathetic function (Task Force, 1996), this effect suggests that emWave treatment resulted in increased parasympathetic augmentation, or less parasympathetic withdrawal, in response to stress.

The current study is one of two studies that have assessed phasic changes during stress in HRV following emWave treatment, and is only one of three to assess such changes within the HRV biofeedback literature as a whole. Another study utilized the Freeze-Frame (prior version of emWave) with children in middle school and found no changes in reactivity to stress (McCraty et al., 1999). HF and LF HRV were examined, but pNN50 was not measured. Therefore, the current finding showing changes in pNN50 reactivity to stress among treatment participants cannot be compared to McCraty et al.'s findings directly. Further, interpretation of 
their results is extremely difficult for several reasons. For example, HRV biofeedback was part of a larger intervention with several other components that may have influenced HRV outcomes, and not all participants received biofeedback as part of the overall treatment.

The only other study offering results for comparison found that participants receiving traditional HRV biofeedback showed increases in pNN50 (hand grip and cold pressor tasks) and LF HRV (hand grip task only) during stress between pre- and post-treatment periods, while the control group did not show increases across time points (Hallman et al., 2011). Therefore, although evidence is limited, the current study and previous findings from Hallman et al. (2011) suggest that HRV biofeedback confers increases in parasympathetic activation in the context of stress. It is possible, then, that HRV biofeedback might effectuate physiological mitigation of the stress response by supporting parasympathetic activity to buffer a strong sympathetic reaction to stress.

Although Hallman and colleagues (2011) also found increases in LF HRV during a hand grip stressor, this finding was not replicated with the cold pressor task in their study, and current results using all study participants did not indicate a change in LF HRV during phasic responses to stress. Phasic changes during stress in LF HRV therefore are not consistently observed. Of note, neither the current results nor those of Hallman and coauthors (2011) showed significant changes in HF HRV or SDNN in reaction to stress. Therefore, changes in parasympathetic activity during stress appears to be susceptible to change following HRV biofeedback treatment, but parallel changes in other HRV parameters appears less likely based on available data.

It should be noted that exploratory analyses of treatment responders in the current study indicated that effects of emWave biofeedback on HRV reactivity may extend beyond the single measure of pNN50. When the two participants who appeared to respond to treatment differently 
than other treatment participants were excluded and main analyses of physiological outcomes were repeated, significant Group by Session interactions emerged for LF HRV, SDNN, and RMSSD during stress. This pattern of results would indicate a more general increase in HRV during stress following emWave intervention, as opposed to an isolated effect on parasympathetic nervous system functioning (i.e., pNN50). No established parameters exist to differentiate treatment responders from non-responders per se, so excluding these two participants could introduce selection bias into the research design. The two participants may represent a subset of individuals whose phasic physiology does not respond to emWave treatment, whereas the majority of participants who received emWave training represented a group that did experience changes in phasic physiology following treatment. This will be an avenue for future studies to pursue. Another possibility is that these participants experienced frustration due to difficulty in improving consistently across sessions, which may have affected their physiological presentation at the post-test assessment. As they engaged in more sessions than the other treatment group members, these two participants also had a longer time period between the pre- and post-test assessment visits. However, this is unlikely to have affected the outcome, as control group participants' assessment visits were yoked with visits of participants in the treatment group on length of time since the initial session.

5.1.3. Heart Rate Variability Recovery. Whereas results of the current study support a treatment effect of the emWave on pNN50 during stress, there were no effects of treatment on recovery from stress for $\mathrm{pNN} 50$, or any other HRV parameter. Therefore, results of the present study do not support the efficacy of emWave treatment in improving physiological restoration of the autonomic nervous system's tonic levels of activity. In their study of implementing HRV biofeedback and other self-regulation treatments in middle schoolers, McCraty and associates 
(1999) found improvements in LF HRV during recovery from stress. As noted above, however, interpretation of the results of this study is difficult due to flaws in study design. Ultimately, no reliable data exists to date indicating efficacy of emWave treatment in improving HRV recovery from stress.

Two traditional HRV biofeedback studies assessed changes in HRV during recovery from stress. One study reported significant increases in HF HRV between periods of stress and recovery during a physical stressor task (Nolan et al., 2005). However, they did not report controlling for resting levels of HRV. Therefore, evidence from this study of improvement in HF HRV during recovery is weak. The results of Hallman et al.'s (2011) study showed increases from pre- to post-treatment for the treatment group in SDNN during recovery from a hand grip stressor, and no changes emerged for the control group. No improvements in SDNN during recovery from the cold pressor task were observed, and improvements were lacking in all other HRV outcomes assessed for recovery from the handgrip and cold pressor stress tasks (i.e., HF HRV, LF HRV, and pNN50).

The current study is the first investigation designed to examine the efficacy of the emWave as a standalone treatment in facilitating improvement in phasic physiological responses to stress. Partial support for its efficacy was garnered in that there was evidence for a treatment effect on parasympathetic responses to stress as measured by pNN50. This finding aligns with outcomes for pNN50 during stress reported in a traditional HRV biofeedback study (Hallman et al., 2011). Therefore, change in parasympathetic activity during stress following HRV biofeedback treatment appears plausible. However, the strength and reliability of this effect is questionable in light of non-significant findings for the other measures of parasympathetic activity (i.e., HF HRV and RMSSD) in this study, and for HF HRV in Hallman et al.'s (2011) 
investigation. If HRV biofeedback conferred a strong and reliable effect on parasympathetic function during stress, change across other indices of parasympathetic function may be expected; however, this has not been borne out consistently in the existing studies. Selection bias aside, the current study does provide some preliminary data supporting a broader increase in multiple HRV measures in response to emWave training among participants who responded to the intervention.

Although changes during the stress response itself may be important in this buffering process, recovery from stress is a critical process as well because extended periods of arousal and related failure to return to physiological homeostasis can also burden the cardiovascular system. Whereas some support was found for changes in stress reactivity following emWave treatment, no evidence was observed in the current study for efficacy of emWave treatment in altering HRV during recovery from stress. Those receiving emWave treatment did not differ from those in the control group in HRV outcomes following stress after completing treatment. Considering the current results within the context of the few existing investigations of changes in recovery from stress following HRV biofeedback treatment, there is little evidence for treatment effects, and inconsistencies are lacking in what little support is available. Therefore, current evidence does not indicate that reliable change in physiological recovery from stress occurs following HRV biofeedback treatment.

Although previous literature examining physiological effects of emWave is limited in number of studies and is wrought with methodological flaws, the traditional HRV biofeedback literature also shows little evidence for long-term changes in HRV as a result of HRV biofeedback treatment. Several of these studies using traditional HRV biofeedback evidence acute changes in LF HRV, as would be expected, during biofeedback practice (see Wheat \& Larkin, 2010). However, lasting physiological changes in tonic HRV generally are not supported 
by current evidence. This study, and results of other studies examining the effects of the emWave, are therefore consistent with the HRV biofeedback literature at large, in that treatment effects on resting HRV are not apparent.

Despite the conception that improvements in HRV may serve to buffer the effects of stress on the cardiovascular system, very little research examining changes in phasic physiological responses to stress existed prior to this study. Further, the only study specifically examining phasic responses following emWave treatment (McCraty et al., 1999) was poorly designed to address that question. The current results add to the small body of literature on phasic responses to stress, indicating possible isolated improvements during stress in parasympathetic responding (i.e., pNN50), and no apparent improvements in recovery from stress. Considering the current results with the pre-existing evidence on changes in phasic responses, the lack of reliable or robust changes in tonic and phasic HRV following HRV biofeedback treatment is striking.

Although anticipating such changes is plausible given the conception that HRV biofeedback may confer clinical improvements in stress-related illnesses and symptoms by altering the stress response, it is possible that HRV biofeedback treatment exhibits clinical efficacy via another mechanism. For example, biofeedback practice may produce physiological effects other than changes in HRV, such as changes in inflammatory processes, which could facilitate improvement in clinical outcomes (Lehrer et al., 2004). Further, some literature supports changes in baroreflex function as a consequence of HRV biofeedback treatment (Lehrer et al., 2003; Lehrer at el., 2004), or baroreflex biofeedback (Reyes del Paso \& Gonzalez, 2004). Baroreflexes also are thought to confer resonant properties on the cardiovascular system and to be exercised during traditional HRV biofeedback (Lehrer, 2007; Vaschillo, Vaschillo, \& Lehrer, 
2006). Given the parallels discussed earlier in this manuscript between biofeedback of the emWave and traditional biofeedback, it is possible that emWave biofeedback exercises the baroreflexes as well. Baroreflex function was not measured directly in the current study, so it is unknown currently whether emWave treatment exercises the baroreflexes and/or strengthens the baroreflex response to stress. However, given that no change in any measure of HRV was detected during emWave treatment sessions, and yet, participants exhibited increased pNN50 during post-treatment stress exposures, it is possible that baroreflexes were being stimulated and exercised during treatment sessions. Consequently, participants who exercised their baroreflexes during training displayed their improved baroreceptor "fitness" following treatments, but only when such reflex action was warranted (i.e., during stress). Therefore, the physiological effects of HRV biofeedback on adaptability of the physiological system to stress may be apparent in baroreflex improvement, rather than in changes in the HRV parameters included in the current investigation.

\subsection{Effects of emWave on Affective Responses to Stress}

State affect in the context of stress was assessed to supplement measures of physiological indices of reactivity and recovery. No significant interactions emerged for any of the MAACL-R subscales. Therefore, there were no apparent treatment effects of the emWave training protocol on affective responses to stress. Emotion regulation is part of the quick coherence technique utilized during the biofeedback sessions by treatment participants. However, participants were instructed not to use any of these methods during the post-treatment assessment as doing so would influence HRV and make discernment of treatment effects on phasic physiological responding impossible. Therefore, emotion regulation strategies utilized during biofeedback sessions should not have translated to the post-treatment session and been apparent in MAACL- 
$\mathrm{R}$ responses. None of the emWave or traditional HRV biofeedback studies implementing stressor protocols assessed affective responses as part of the stressor protocols, so comparisons to previous investigations were not possible.

\subsection{Effects of emWave on Stress and Psychological Symptom Distress}

Most studies examining the effects of emWave treatment focused on non-physiological outcomes. Therefore, self-report measures of general stress and distress secondary to psychological symptoms were included for purposes of supplementing physiological outcome indices.

No interactions emerged for scores on the BSI or any subscales of the PSS to demonstrate that emWave treatment affected psychological symptom distress or overall self-reported stress levels. The main effects of session (i.e., the Global Severity Index of the BSI, the Anxiety and Somatization subscales of the BSI, and PSS scores reduced across sessions in both groups) may reflect regression toward the mean. Due to the absence of an interaction between group and session, these observed reductions within the treatment group cannot be attributed to emWave treatment effects.

Several investigations of effects of emWave (or Freeze-Framer) treatment on stress and mental health outcomes have been conducted (e.g., Barrios-Choplin et al., 1999; Climov, 2008; McCraty, Atkinson, Lipsenthal, \& Arguelles, 2003; McCraty et al., 2003b; McCraty, Atkinson, Tomasino, Goelitz, \& Mayrovitz, 1999; McCraty, Tomasino, Atkinson, Aasen, \& Thurik, 2000). Each of these studies evidenced some change, if not several changes, in psychological parameters from pre- to post-treatment, and changes were in anticipated directions (i.e., reductions in symptom levels). Unfortunately, aspects of the studies were often too poorly planned or controlled (e.g., lack of control group, inconsistent application of biofeedback across 
participants, development of a measure without appropriate of validity/reliability assessment) to make meaningful conclusions regarding these changes viable. Further, the findings tended to be inconsistent across studies when the same measures were used across multiple investigations.

Several peer-reviewed studies on the effects of HRV biofeedback training included selfreport measurement of related constructs. One of these studies utilized emWave treatment, and decreases were evident across groups in anxiety and general distress as measured by the StateTrait Anxiety Inventory and the Mood and Anxiety Symptom Questionnaire (Henriques et al., 2011). However, there were no interaction effects to demonstrate a treatment effect specific to the emWave treatment protocol. Several traditional HRV biofeedback studies have also provided evidence for decreases in reports of anxiety (Hospital Anxiety and Depression Scale) and/or depression (Beck Depression Inventory II; Hamilton Rating Scale for Depression; Hospital and Anxiety Depression Scale), but did not include a control group to allow the conclusion that these reductions were due to treatment effects (e.g., Giardino, Chan, \& Borson, 2004; Hassett et al., 2007; Karavidas et al., 2007). Other studies including control groups also found main effects for time or session across groups on reports of depression (Center for Epidemiologic Studies Depression Scale), stress (PSS), and/or positive and negative affect (Positive and Negative Affect Schedule), while failing to find interaction effects that would suggest a HRV biofeedback treatment effect (Nolan et al., 2005; Swanson et al., 2009). One study examining traditional HRV biofeedback reported a significant interaction between group and time wherein reports of depression were decreased to a higher degree in treatment participants than in control participants (Zucker et al., 2009).

In general, current results do not provide much evidence that emWave treatment reduces reports of stress or psychological distress across various domains (e.g., depression, anxiety), 
which is consistent with the majority of findings of other HRV biofeedback studies. Further, the main effects for time across both groups on PSS and some BSI subscales appears to be consistent with prior literature showing similar patterns of main effects, without a differential effect of treatment on these outcomes.

\subsection{Study Limitations}

Like all intervention research, this study is not without limitations. The emWave program was not designed for use in clinical trials and does not have an established protocol. Although those who developed the emWave program provided a clinician guide offering some recommended parameters for treatment delivery (Culbert et al., 2007), no evidence-based information regarding implementation of the emWave treatment is available to guarantee appropriate length of treatment, method for documenting progression in treatment, the necessary components to include in treatment, etc. Parameters for the current study were established based to some extent on clinical guidelines provided with the emWave clinician guide (e.g., minimum of 4 sessions). Other details were implemented to promote standardization across participants at the discretion of the author in the absence of empirical indication regarding standards of practice. It is possible that analyses for the current study did not reveal significant effects because the method of treatment delivery utilized for this study did not establish a sufficiently strong treatment effect. However, given that specific instructions for implementing training were not available, the protocol employed was at least congruent with recommended training procedures. It is possible, however, that training that lasted longer or employed a higher criterion for completion may have resulted in differential effects.

It also is possible that changes in tonic or phasic LF HRV, HF HRV, SDNN, pNN50, and RMSSD do not measure the mechanism through which HRV biofeedback exerts its clinical 
effect. Changes in these parameters have not been commonly revealed in traditional HRV biofeedback studies. Although the current study is among the initial empirical efforts to determine the effect of emWave treatment on tonic and phasic measures of HRV, the null findings are consistent with previous empirical work employing standard HRV biofeedback protocols. Improvements in baroreflex functioning may be a viable mechanism through which HRV biofeedback confers clinical improvement across medical and psychological symptoms (see Wheat \& Larkin, 2010). As baroreflex functioning was not measured in the current study, an additional limitation of this investigation is that the effect of emWave treatment on this parameter cannot be explored. No other emWave study has measured baroreflex sensitivity or gain, so the ways in which their activity may be improved by emWave treatment cannot be determined at this time.

As the primary aim of this study was to examine physiological changes in response to emWave treatment, healthy volunteers were recruited for participation to eliminate potentially confounding disease states and interference by medications less health participants may have been prescribed. In this regard, the magnitude of treatment effects may have been negligible due to a ceiling effect, whereby participants already tended to exhibit adequate physiological responses to stress. A stronger likelihood of altering one's physiological responses to stress may be observed among clinical samples where HRV and associated parameters are known to be compromised. Future research will be needed to evaluate the utility of altering one's physiological responses to stress via HRV biofeedback among patients with chronic medical or psychiatric conditions.

The current study was the first to assess affective reactivity during a stressor protocol following emWave treatment. The MAACL-R was utilized for this purpose, and internal 
consistency was low for some subscales of this instrument. In general, internal consistency of these scales has been established in other studies. The small sample size and low variance in affective responding among participants in the current study likely contributed to the poorer levels of internal consistency observed. Therefore, effect of emWave treatment on affective responses to stress could not be assessed with complete confidence.

An additional limitation of this study is that respiration was not measured concurrent to either assessment or training sessions and may have accounted for the relative lack of significant findings associated with biofeedback treatment. Courtney et al. (2011) illustrated that some individuals may exhibit dysfunctional breathing patterns that could affect their ability to improve HRV and obtain coherence during biofeedback. Individuals with these types of irregular breathing patterns may undergo treatment, but fail to optimize effects on HRV or exercise baroreflexes in the process. Therefore, if treatment effects are mediated by physiological changes that occur during biofeedback, the breathing abnormalities of these individuals could obscure treatment outcomes. As breathing patterns were not measured in this study, determining whether breathing dysfunctions existed among study participants was not determined, which may have affected results. For example, it is possible that the two participants excluded for the purposes of exploratory analyses showed these breathing abnormalities. The full analyses may have been tempered by inclusion of their data, thereby yielding largely non-significant results.

Further, participants were instructed to engage in utilizing the quick coherence technique independently, without the aid of biofeedback, each day between session visits. Although they logged practice, participants' reports of practice may have been inaccurate or exaggerated, and practice may not have been physiologically adequate without the presence of biofeedback. As 
these factors may have contributed to the low level of treatment effects noted, this also is a relative weakness of the current study.

Finally, a few aspects of the current study limit the generalizability of findings. First, our sample did not exhibit an even gender distribution. Although previous HRV biofeedback studies have not shown that males and females respond differently to relevant interventions, results of this study cannot be generalized to males due to their limited presence in this sample. Our sample may also be unique in that the participants were recruited from psychology courses and/or programs of study. Participants may have had more familiarity than individuals not formally educated in psychology with behavioral interventions for psychiatric and/or medical symptoms or illnesses, and it is possible that they may have been more amenable to engaging readily in emWave treatment for that reason. Additional evidence for the unique attributes of the study sample pertain to the finding that several HRV parameters, particularly those occurring during stress presentations, were positively correlated with participant age. This suggests that the older participants who were graduate students in psychology had greater HRV, indicating a somewhat healthier stress response than the younger undergraduate participants. Given the number of years graduate students have studied the science of psychology, they may have had a greater opportunity to use their knowledge to develop more effective stress management skills than undergraduate students. Alternatively, because the experimenter of the current study was a graduate student in psychology, graduate student participants may have simply been less stressed while engaging in the tasks for a peer than undergraduates performing for someone who they perceive as an authority figure. Regardless of the reason for this finding, age was covaried in all analyses to remove any variance accounted for by participant age. 


\subsection{Future Directions}

Due to the paucity of peer-reviewed literature on effects of emWave treatment, there are several areas in which the literature may be improved. Within the context of HRV biofeedback in general, the current study also provides information for areas of improvement in that broader literature as well.

As noted above, although the current study examined the effects of emWave in healthy adults, examining the efficacy of emWave biofeedback in clinical populations in one avenue for future studies to address. It will be informative to extend the use of emWave in clinical populations to determine whether it may prove to be efficacious in producing physiological and/or clinical changes in those with psychiatric or medical complaints, as well as in those who exhibit affected physiology due to related conditions. An additional population that may benefit from emWave treatment is individuals involved in athletics. Prior investigations illustrate the utility of HRV biofeedback in improving performance in various athletic groups (e.g., Lagos, Vaschillo, Vaschillo, Lehrer, Bates, \& Pandina, 2011; Raymond, Sajid, Parkinson, \& Gruzelier, 2005; Strack, 2003; Vaschillo, Vysochin, \& Rishe, 1998). It is possible that the emotional and physiological regulation targeted by emWave treatment could improve attention, reaction time, performance, etc., in athletes in an accessible, affordable, and easily implemented format. Future studies may target athletes a special population in which to test the efficacy of the emWave.

This is the second HRV biofeedback study, and the first utilizing the emWave, that found an effect of treatment on pNN50 during reactivity to stress (Hallman et al., 2011). As this effect on parasympathetic responding during stress has been noted across a healthy sample (current study) and a sample of participants with pain (Hallman et al., 2011), as well as across two different modalities of HRV biofeedback, it may represent a reliable treatment effect. Replication 
of this finding would be beneficial to determine if comparable effects can be noted across additional populations to which HRV biofeedback treatment is delivered. This is one promising avenue through which emWave treatment, or HRV biofeedback treatment in general, may confer a change in the physiological stress response. Investigators may also consider examining in a larger-scale study whether changes also occur in other HRV parameters, as exploratory analyses excluding two treatment participants evidenced a broader spectrum of changes in phasic HRV responses (i.e., pNN50 in addition to LF HRV, SDNN, and RMSSD). Our exploratory approach suggests that emWave intervention may confer general increases in HRV during stress, but this cannot be concluded with certainty at the current time.

Based on the larger biofeedback literature, baroreflex function may be a good candidate to consider when searching for the mechanism through with HRV biofeedback has exerted an influence on clinical outcomes. No emWave studies have implemented measurement of baroreceptor or baroreflex function, and a limited number of traditional HRV biofeedback studies have done so. In light of unreliable or non-existent chronic changes in measures of HRV (excepting the findings on pNN50 reactivity change with emWave noted above), increasing attention to baroreflex measures in subsequent investigations may help elucidate the mechanism involved in producing clinical changes following HRV biofeedback.

Ultimately, to rigorously assess the efficacy and effectiveness of emWave treatment as a viable way to treat emotional and physical disorders, a standardized treatment approach should be developed for use in empirical assessments of the treatment. A stage model for behavioral interventions (Rounsaville, Carroll, \& Onken, 2001) offers guidelines on systematic efforts to build toward clinical trials that may establish empirically supported treatments. This study represents an effort to contribute to the first stage of those efforts, but much more empirical work 
is necessary to progress beyond early stages toward full-fledged clinical trials. For example, investigations addressing treatment development, manual development, specification of session content, determination of treatment length, etc. will assist in maximizing the potential for efficacy of treatment and, importantly, testability of emWave treatment as such.

In a related vein, efforts should be made to evaluate components of emWave treatment in peer-reviewed arenas systematically. One recent study (Henriques et al., 2011) is a commendable example of subjecting the emWave treatment protocol to the same rigorous standards of empirical examination that have been applied to traditional HRV biofeedback treatment. Much investigation of emWave treatment (and its predecessor, Freeze-Frame) has been unsystematic (e.g., lack of control group, failure to adequately define treatment components, unequal delivery of treatment across participants), and some studies have not undergone the peer review process. Systematic development and investigation by third party researchers are warranted to truly determine the potential of emWave treatment as an efficacious approach to managing psychiatric or medical symptoms.

\section{Conclusions}

Given the ease of use and relatively low cost of the emWave, continuing to investigate its effects to establish whether it reliably produces either physiological or clinical change is warranted. The current study documented one way in which emWave treatment affected a limited aspect of phasic HRV responses to stress and, in doing so, replicated one other study finding a similar effect. Based on the understanding that HRV biofeedback may confer clinical improvements in disease states as documented in the literature, it is somewhat surprising that a bigger impact on physiology was not achieved in the current study. However, this appears to be an issue present in the traditional HRV biofeedback literature at large as well. The scientific and 
clinical communities will benefit from broadening our conceptualization of ways in which HRV biofeedback may confer meaningful clinical changes and, in turn, move us forward to establish which modalities address this mechanism most efficiently and which populations may benefit to the greatest degree. 


\section{References}

Agelink, M., Boz, C., Ullrich, H., \& Andrich, J. (2002). Relationship between major depression and heart rate variability: Clinical consequences and implications for antidepressive treatment. Psychiatry Research, 113, 139-149.

Akselrod, S., Gordon, D., Ubel, F. A., Shannon, D.C., Berger, A. C., Cohen, R. J. (1981). Power spectrum analysis of heart rate fluctuation: A quantitative probe of beat-to-beat cardiovascular control. Science, 213, 220-222.

Amon, K., \& Campbell, A. (2008). Can children with AD/HD learn relaxation and breathing techniques through biofeedback video games? Australian Journal of Educational \& Developmental Psychology, 8, 72-84.

Barrios-Choplin, B., McCraty, R., \& Cryer, B. (1997). An inner quality approach to reducing stress and improving physical and emotional wellbeing at work. Stress Medicine, 13, 193-201.

Barrios-Choplin, B., McCraty, R., Sundram, J., \& Atkinson, M. (1999). The effect of employee self-management training on personal and organizational quality. Retrieved from http://www.heartmath.org/research/publications.html.

Berntson, G., Bigger, T., Eckberg, D., Grossman, P., Kaufmann, P., Malik, M., et al. (1997). Heart rate variability: Origins, methods, and interpretive caveats. Psychophysiology, 34, 623-648.

Bigger, J., Fleiss, J., Rolnitzky, L., \& Steinman, R. (1993). The ability of several short-term measures of RR variability to predict mortality after myocardial infarction. Circulation, $88,927-934$. 
Bonnemeier, H., Wiegand, E., Brandes, A., Kluge, N., Katus, H., Richardt, G., \& Potratz, J. (2003). Circadian profile of cardiac autonomic nervous modulation in healthy subjects: differing effects of aging and gender on heart rate variability. Journal Of Cardiovascular Electrophysiology, 14(8), 791-799.

Boysen, A., Lewin, M., Hecker, W., Leichter, H., \& Uhlemann, F. (2007). Autonomic function testing in children and adolescents with diabetes mellitus. Pediatric Diabetes, 8, 261-264.

Carpeggiani, C., L'Abbate, A., Landi, P., Michelassi, C., Raciti, M., Macerata, A., et al. (2004). Early assessment of heart rate variability is predictive of in-hospital death and major complications after acute myocardial infarction. International Journal of Cardiology, 96, $361-368$.

Climov, D. (2008). Results of a stress management program for graduate students based on relaxation associated with HRV biofeedback. Retrieved from http://www.heartmath.org/research/publications.html.

Cohen, H., Benjamin, J., Geva, A., Matar, M., Kaplan, Z., \& Kotler, M. (2000). Autonomic dysregulation in panic disorder and in post-traumatic stress disorder: Application of power spectrum analysis of heart rate variability at rest and in response to recollection of trauma or panic attacks. Psychiatry Research, 96, 1-13.

Cohen, S., Kamarck, T., \& Mermelstein, R. (1983). A global measure of perceived stress. Journal of Health and Social Behavior, 24, 385-396.

Cohen, H., Kotler, M., Matar, M., \& Kaplan, Z. (1997). Power spectral analysis of heart rate variability in posttraumatic stress disorder patients. Biological Psychiatry, 41, 627-629. 
Correia, D., Rodrigues De Resende, L., Molina, R., Ferreira, B., Colombari, F., Barbosa, C., et al. (2006). Power spectral analysis of heart rate variability in HIV-infected and AIDS patients. Pacing \& Clinical Electrophysiology, 29, 53-58.

Cowan, M. Pike, K., \& Budzynski, H. (2001). Psychosocial nursing therapy following sudden cardiac arrest: Impact on two-year survival. Nursing Research, 50, 68-76.

Culbert, T.P., Martin, H., \& McCraty, R. (2007). A practitioner's guide: Applications of the emWave PC Stress Relief System. HeartMath, LLC. Boulder, CO.

Del Pozo, J., Gevirtz, R., Scher, B., \& Guarnert, E. (2004). Biofeedback treatment increases heart rate variability in patients with known coronary artery disease. American Heart Journal, 147, G1-G6.

Derogatis, L. R. (2001). Brief Symptom Inventory (BSI) 18: Administration, scoring, and procedures manual. Minneapolis, MN: NCS Pearson.

Derogatis, L., \& Melisaratos, N. (1983). The Brief Symptom Inventory: An introductory report. Psychological Medicine, 13, 595-605.

Felber Dietrich, D., Schindler, C., Schwartz, J., Barthelemy, J., Tschopp, J., Roche, F., et al. (2006). Heart rate variability in an aging population and its association with lifestyle and cardiovascular risk factors: Results of the SAPALDIA study. Europace 8, 521-529.

Giardino, N., Chan, L., \& Borson, S. (2004). Combined heart rate variability and pule oximetry biofeedback for chronic obstructive pulmonary disease: Preliminary findings. Applied Psychophysiology \& Biofeedback, 29, 121-133.

Goodie, J., Larkin, K., \& Schauss, S. (2000). Validation of Polar heart rate monitor for assessing heart rate during physical and mental stress. Journal of Psychophysiology, 14, 159-164. 
Griffin, M., Lake, D., Bissonette, E., Harrell Jr., F., O'Shea, T., \& Moorman, J. (2005). Heart rate characteristics: Novel physiomarkers to predict neonatal infection and death. Pediatrics, $116,1070-1074$.

Hallman, D., Olsson, E., von Scheele, B., Melin, L., Lyskov, E. (2011). Effects of heart rate variability biofeedback in subjects with stress-related chronic neck pain: A pilot study. Applied Psychophysiology and Biofeedback, 36, 71-80.

Hassett, A., Radvanski, D., Vaschillo, E., Vaschillo, B., Sigal, L., Karavidas, M., et al. (2007). A pilot study of the efficacy of heart rate variability (HRV) biofeedback in patients with fibromyalgia. Applied Psychophysiology \& Biofeedback, 32, 1-10.

Heilman, K., Handelman, M., Lewis, G., \& Porges, S. (2008). Accuracy of the StressEraser ${ }^{\circledR}$ in the detection of cardiac rhythms. Applied Psychophysiology \& Biofeedback, 33, 83-89.

Henriques, G., Keffer, S., Abrahamson, C., Horst, S. J. (2011). Exploring the effectiveness of a computer-based heart rate variability biofeedback program in reducing anxiety in college students. Applied Psychophysiology and Biofeedback, 36, 101-112.

Karavidas, M., Lehrer, P., Vaschillo, E., Vaschillo, B., Marin, H., Buyske, S., et al. (2007). Preliminary results of an open label study of heart rate variability biofeedback for the treatment of major depression. Applied Psychophysiology \& Biofeedback, 32, 19-30.

Kim, C., McGorray, S., Bartholomew, B., Marsh, M., Dicken, T., Wassertheil-Smoller, S., et al. (2005). Depressive symptoms and heart rate variability in postmenopausal women. Archives of Internal Medicine, 165, 1239-1244.

Kuo, T., Lin, T., Yang, C., Li, C., Chen, C., \& Chou, P. (1999). Effect of aging on gender differences in neural control of heart rate. American Journal of Physiology - Heart and Circulatory Physiology, 277, 2233-2239. 
Lagos, L., Vaschillo, E., Vaschillo, B., Lehrer, P., Bates, M., \& Pandina, R. (2011). Virtual reality-assisted heart rate variability biofeedback as a strategy to improve golf performance: A case study. Biofeedback, 39, 15-20.

La Rovere, M., Bigger Jr., J., Marcus, F., Mortara, A., \& Schwartz, P. (1998). Baroreflex sensitivity and heart-rate variability in prediction of total cardiac mortality after myocardial infarction. Lancet, 351, 478-484.

LeFevers, V. (1971). Volitional control of heart rate during exercise stress. (ERIC Document Reproduction Service No. ED088793).

Lehrer, P. (2007). Biofeedback training to increase heart rate variability. In P. M. Lehrer, R. L. Woolfolk, \& W. E. Sime (Eds.). Principles and practice of stress management (3rd ed.) (227-248). New York: The Guilford Press.

Lehrer, P., Carr, R., Smetankine, A., Vaschillo, E., Peper, E., Porges, S., et al. (1997). Respiratory sinus arrhythmia versus neck/trapezius EMG and incentive inspirometry biofeedback for asthma: A pilot study. Applied Psychophysiology and Biofeedback, 22, 95-109.

Lehrer, P., Vaschillo, E., Lu, S., Eckberg, D., Vaschillo, B., Scardella, A., et al. (2006). Heart rate variability biofeedback: Effects of age on heart rate variability, baroreflex gain, and asthma. Chest, 129, 278-284.

Lehrer, P., Vaschillo, E., \& Vaschillo, B. (2000). Resonant frequency biofeedback training to increase cardiac variability: Rationale and manual for training. Applied Psychophysiology \& Biofeedback, 25, 177-191. 
Lehrer, P., Vaschillo, E., Vaschillo, B., Lu, S., Eckberg, D., Edelberg, R., et al. (2003). Heart rate variability biofeedback increases baroreflex gain and peak expiratory flow. Psychosomatic Medicine, 65, 796-805.

Lehrer, P., Vaschillo, E., Vaschillo, B., Lu, S., Scardella, A., Siddique, M., et al. (2004). Biofeedback treatment for asthma. Chest, 126, 352-361.

Lerner, J., Dahl, R., Hariri, A., \& Taylor, S. (2007). Facial expressions of emotion reveal neuroendocrine and cardiovascular stress responses. Biological Psychiatry, 61, 253-260.

Lubin, B., Van Whitlock, R., Reddy, D., Petren, S. (2001). A comparison of the short and long forms of the multiple affect adjective checklist - revised. Journal of Clinical Psychology, 57, 411-416.

Luskin, F., Reitz, M. Newell, K., Quinn, T. G., Haskell, W. (2002). A controlled pilot study of stress management training of elderly patients with congestive heart failure. Preventative Cardiology, 5, 168-172.

Martínez-Lavín, M., Hermosillo, A., Rosas, M., \& Soto, M. (1998). Circadian studies of autonomic nervous balance in patients with fibromyalgia: A heart rate variability analysis. Arthritis and Rheumatism, 41, 1966-1971.

McCanne, T., \& Sandman, C. (1974). Instrumental heart rate responses and visual perception: A preliminary study. Psychophysiology, 11, 283-287.

McCraty, R., Atkinson, M., Lipsenthal, L., \& Arguelles, L. (2003a). Impact of the power to change performance program on stress and health risks in correctional officers. Retrieved from http://www.heartmath.org/research/publications.html. 
McCraty, R., Atkinson, M., \& Tomasino, D. (2003b). Impact of a workplace stress reduction program on blood pressure and emotional health in hypertensive employees. Journal of Alternative \& Complementary Medicine, 9, 355-369.

McCraty, R., Atkinson, M., Tomasino, D., \& Bradley, R. T. (2006). The coherent heart: Heartbrain interactions, Psychophysiological coherence, and the emergence of system-wide order. Retrieved from http://www.heartmath.org/research/publications.html.

McCraty, R., Atkinson, M., Tomasino, D., Goelitz, J., \& Mayrovitz, H. N. (1999). The impact of an emotional self-management skills course on psychosocial functioning and autonomic recovery to stress in middle school children. Integrative Physiological and Behavioral Science, 34, 246-268.

McCraty, R., Barrios-Choplin, B., Rozman, D., Atkinson, M., \& Watkins, A. D. (1998). The impact of a new emotional self-management program on stress, emotions, heart rate variability, DHEA and cortisol. Integrative Physiological and Behavioral Science, 33, 151-170.

McCraty, R., Tomasino, D., Atkinson, M., Aasen, P., \& Thurik, S. J. (2000). Improving testtaking skills and academic performance in high school students using HeartMath learning enhancement tools. Retrieved from http://www.heartmath.org/research/publications.html.

McGrady, A. (2007). Psychophysiological mechanisms of stress: A foundation for the stress management therapies. In P. M. Lehrer, R. L. Woolfolk, \& W. E. Sime (Eds.). Principles and practice of stress management (3rd ed.) (16-37). New York: The Guilford Press.

Muench, F. (2008). The portable Stress Eraser heart rate variability biofeedback device: Background and research. Biofeedback, 36, 35-39. 
Nolan, R., Kamath, M., Floras, J., Stanley, J., Pang, C., Picton, P., et al. (2005). Heart rate variability biofeedback as a behavioral neurocardiac intervention to enhance vagal heart rate control. American Heart Journal, 149, 1137.e1-1137.e7.

Phelps, E.A. (2006). Emotion and cognition: Insights from studies of the human amygdala. Annual Review of Psychology, 57, 27-53.

Pinna, G., Maestri, R., Torunski, A., Danilowicz-szymanowicz, L., Szwoch, M., La Rovere, M. T., Raczack, G. (2007). Heart rate variability measures: A fresh look at reliability. Clinical Science, 113, 131-140.

Raymond, J., Sajid, I., Parkinson, L. A., \& Gruzelier, J. H. (2005). Biofeedback and dance performance: A preliminary investigation. Applied Psychophysiology and Biofeedback, $30,65-73$.

Reyes del Paso, G., \& Gonzalez, M. (2004). Modification of baroreceptor cardiac reflex function by biofeedback. Applied Psychophysiology and Biofeedback, 29, 197-211.

Rounsaville, B. J., Carroll, K. M., \& Onken, L. S. (2001). A stage model of behavioral therapies research: Getting started and moving on from stage I. Clinical Psychology: Science And Practice, 8(2), 133-142.

Rozman, D., Whitaker, R., Beckman, T., \& Jones, D. (1996). A pilot intervention program which reduces psychological symptomology in individuals with human immunodeficiency virus. Complementary Therapies in Medicine, 4, 226-232.

Schmidt, L., Fox, N., Perez-Edgar, K., \& Hamer, D. (2009). Linking gene, brain, and behavior: DRD4, frontal asymmetry, and temperament. Psychological Science, 20, 831-837.

Schoenfeld, W., Matos, M., \& Snapper, A. (1967). Cardiac conditioning in the white rat with food presentation as unconditional stimulus. Conditional Reflex, 2, 56-67. 
Schroeder, E., Liao, D., Chambless, L., Prineas, R. Evans, G., \& Heiss, G. (2003). Hypertension, blood pressure, and heart rate variability: The Atherosclerosis Risk in Communities (ARIC) study. Hypertension, 42, 1106-1111.

Schulz, K. F., Altman, D. G., \& Moher, D. for the CONSORT Group. (2010) CONSORT 2010 statement: Updated guidelines for reporting parallel group randomized trials. Annals of Internal Medicine, 152, 1-8.

Schwartz, N., \& Schwartz, M. (2003). Definitions of biofeedback and applied psychophysiology. In M. S. Schwartz \& F. Andrasik (Eds.), Biofeedback: A practitioner's guide (3rd ed.) (27-39). New York: The Guilford Press.

Shaffer, F., Neblett, R., \& Crawford, J. (2008). Biofeedback Certification Institute of America plans for the future with you in mind. Biofeedback, 36, 9-10.

Shea, A., Kamath, M., Fleming, A., Streiner, D., Redmond, K., \& Steiner, M. (2008). The effect of depression on heart rate variability during pregnancy. Clinical Autonomic Research, $18,203-212$.

Strack, B. W. (2003). Effect of heart rate variability (HRV) biofeedback on batting performance in baseball. Dissertation Abstracts International: Section B: The Sciences and Engineering, 64, 1540.

Swanson, K. S., Gevirtz, R. N., Brown, M., Spira, J. Guarneri, E., \& Stoletny, L., (2009). The effect of biofeedback on function in patients with heart failure. Applied Psychophysiology and Biofeedback, 34, 71-91.

Task Force of the European Society of Cardiology and the North American Society of Pacing and Electrophysiology. (1996). Heart rate variability: Standards of measurement, physiological interpretation and clinical use. Circulation, 93, 1043-1065. 
Taylor, S., Burklund, L., Eisenberger, N., Lehman, B., Hilmert, C., \& Lieberman, M. (2008). Neural bases of moderation of cortisol stress responses by psychosocial resources. Journal of Personality \& Social Psychology, 95, 197-211.

Thayer, J., Friedman, B., \& Borkovec, T. (1996). Autonomic characteristics of generalized anxiety disorder and worry. Biological Psychiatry, 39, 255-266.

Vaschillo, E., Vaschillo, B., \& Lehrer, P. (2006). Characteristics of Resonance in Heart Rate Variability Stimulated by Biofeedback. Applied Psychophysiology \& Biofeedback, 31, $129-142$.

Watkins, L., Blumenthal, J., \& Carney, R. (2002). Association of anxiety with reduced baroreflex cardiac control in patients after acute myocardial infarction. American Heart Journal, 143, 460-466.

Yeragani, V., Rao, K., Smitha, M., Pohl, R., Balon, R., \& Srinivasan, K. (2002). Diminished chaos of heart rate time series in patients with major depression. Biological Psychiatry, $51,733-749$.

Zucker, T. L., Samuelson, K. W., Muench, F., Greenberg, M. A., \& Gevirtz, R. N. (2009). The effects of respiratory sinus arrhythmia biofeedback on heart rate variability and posttraumatic stress disorder symptoms: A pilot study. Applied Psychophysiology and Biofeedback, 34, 135-143.

Zuckerman, M., \& Lubin, B. (1985). Manual for the multiple affect adjective checklist. San Diego, CA: Educational and Industrial Testing Service. 
8. Appendices 


\section{Appendix A}

Your age

Your sex
o Male
$\circ$ Women

Your race
$\circ$ Black
$\circ$ White
$\circ$ Hispanic
○ Native American
$\circ$ Asian
○ Biracial (specify):
o Other

Total years of education you have completed:

$\circ$ High school

$\circ 1$ year college

- 2 years college

○ 3 years college

○ 4 or more years college

Please describe any cardiovascular related illness that you may have, including high blood pressure:

Please list any other medical or psychiatric problems that you have:

Please list any major surgeries and medical, or psychiatric illnesses you have had in the past.

Females: When did you start your last menstrual cycle?
o I am a Male
$\circ$ less than one week ago
o one week ago
o two weeks ago
o three weeks ago
$\circ$ four weeks ago
$\circ$ more than four weeks ago
- I am currently pregnant 
Females: Are you currently on birth control (contraceptives).

o I am a male

$\circ$ No

$\circ$ Yes

What type of birth control are you taking?

Please list any drugs (legal or otherwise) that you are currently taking including; birth control (contraceptives), heart medications, cold or allergy medications, over the counter medications, asthma medications, Beta-Blockers (i.e. Inderal, Tenormin), psychoactive drugs (i.e. Adderall, Xanax, Haldol, Lithium, Prozac), or diet pills.

On average, how often do you smoke cigarettes?

o never

○ I am not currently smoking

$\circ$ less than one pack per day

- 1-2 packs per day

- 2-3 packs per day

$\circ$ greater than 3 packs per day

On average, how often do you use smokeless tobacco?

o never

○ I am not currently using smokeless tobacco

$\circ$ 1-4 times per day

○ 5-8 times per day

- 9-13 times per day

$\circ$ greater than thirteen times per day

How often do you drink alcohol?

o never

$\circ$ infrequently (a few drinks per year)

o occasionally (1-2 drinks per month)

○ weekly (1-3 drinks per week)

o weekly (4-7 drinks per week)

$\circ$ daily (7-14 drinks per week)

o daily (more than 14 drinks per week) 
How many cups of caffeinated coffee, tea, or soda do you have per day?
o none
$\circ$ 1-2 cups per day
- 3-4 cups per day
- 5-6 cups per day
$\circ$ 7-8 cups per day
$\circ$ greater than eight cups per day

How many times per week do you engage in aerobic physical activity?
o never
○ 1-2 times
○ 3-6 times
○ 7 or more times

For how long do you typically exercise on each occasion?
$\circ$ 5-10 minutes
○ 11-15 minutes
○ 16-30 minutes
- 31-60 minutes
○ 61-90 minutes
o more than 90 minutes

How many hours of sleep did you get last night?
o Less than 4 hours
o 4-5 hours
० 5-6 hours
○ 6-7 hours
$\circ$ 7-8 hours
o 8-9 hours
$\circ$ greater than 9 hours

Family Information:

What is your best estimate of your family's total income?
$\circ$ Less than 24,999
○ 25,000 to 34,999
○ 35,000 to 49,999
○ 50,000 to 74,999
○ 75,000 to 99,999
○ 100,000 to 149,999
o Greater than 150,000 
How difficult was it for your parents to make their bill payments when you were young?

o Not difficult at all

○ Somewhat difficult (missed a few payments, but rarely)

$\circ$ Very difficult (often missed payments)

$\circ$ Extremely difficult (could rarely make all their payments and had to choose what bills to pay week-to-week)

Approximately how old is your biological father?

If you are adopted, mark here and report on your biological father or move to the section on your mother's health

What is your father's highest level of education

- Some High School

$\circ$ Graduated High School

- Some College

- Graduated College

○ Some Graduate School

- Obtained Masters Degree

- Obtained Doctoral Degree

What is your father's occupation?

Is he currently living?

$\circ$ yes

Did/does your father have high blood pressure (hypertension)?

$\circ$ yes

o no

How certain are you that he did, or did not, have high blood pressure (hypertension)?

o Absolutely (100\%) certain

- Almost (75\%) certain

$\circ$ Not sure at all $(25 \%)$

$\circ$ No information by which to judge $(0 \%)$

Did/does your father have any heart problems such as angina (chest pains), a heart attack, or coronary heart disease?

$\circ$ yes

$\circ$ no

If yes, please specify if you are able: 
How certain are you that he did, or did not, have a heart problem as indicated above?

o Absolutely $(100 \%)$ certain

- Almost (75\%) certain

$\circ$ Not sure at all $(25 \%)$

$\circ$ No information by which to judge $(0 \%)$

$\mathrm{Did} /$ does your father have diabetes?

$\circ$ yes

o no

How certain are you that he did, or did not, have diabetes?

o Absolutely (100\%) certain

o Almost (75\%) certain

$\circ$ Not sure at all $(25 \%)$

$\circ$ No information by which to judge $(0 \%)$

Did/does your father have a kidney disease (other than kidney stones)?

$\circ$ yes

o no

How certain are you that he did, or did not, have a kidney disease (other than kidney stones)?

o Absolutely (100\%) certain

○ Almost (75\%) certain

$\circ$ Not sure at all $(25 \%)$

$\circ$ No information by which to judge $(0 \%)$

Did/does your father have cancer?

$\circ$ yes

$\circ$ no

How certain are you that he did, or did not, have cancer?

○ Absolutely (100\%) certain

○ Almost $(75 \%)$ certain

$\circ$ Not sure at all $(25 \%)$

$\circ$ No information by which to judge $(0 \%)$

If you know, please list what type(s) of cancer he had.

Approximately how old is your biological mother?

If you are adopted, mark here ___ and report on your biological mother 
What is your mother's highest level of education

- Some High School

- Graduated High School

○ Some College

- Graduated College

- Some Graduate School

- Obtained Masters Degree

o Obtained Doctoral Degree

What is your mother's occupation?

Is she currently living?

$$
\begin{aligned}
& \circ \text { yes } \\
& \circ \text { no }
\end{aligned}
$$

Did/does your mother have high blood pressure (hypertension)?

$$
\begin{aligned}
& \circ \text { yes } \\
& \circ \text { no }
\end{aligned}
$$

How certain are you that she did, or did not, have high blood pressure (hypertension)?

o Absolutely (100\%) certain

○ Almost $(75 \%)$ certain

$\circ$ Not sure at all $(25 \%)$

$\circ$ No information by which to judge $(0 \%)$

Did/does your mother have any heart problems such as angina (chest pains), a heart attack, or coronary heart disease?

$\circ$ yes

o no

If yes, please specify if you are able:

How certain are you that she did, or did not, have a heart problem as indicated above?

○ Absolutely (100\%) certain

○ Almost (75\%) certain

- Not sure at all $(25 \%)$

$\circ$ No information by which to judge $(0 \%)$

$\mathrm{Did} /$ does your mother have diabetes?

$\circ$ yes

o no

How certain are you that she did, or did not, have diabetes?

- Absolutely (100\%) certain

○ Almost (75\%) certain

$\circ$ Not sure at all (25\%)

$\circ$ No information by which to judge $(0 \%)$ 
Did/does your mother have a kidney disease (other than kidney stones)?
$\circ$ yes
$\circ$ no

How certain are you that she did, or did not, have a kidney disease (other than kidney stones)?

- Absolutely (100\%) certain

○ Almost (75\%) certain

$\circ$ Not sure at all (25\%)

○ No information by which to judge $(0 \%)$

Did/does your mother have cancer?

$\circ$ yes

o no

How certain are you that she did, or did not, have cancer?

o Absolutely (100\%) certain

○ Almost (75\%) certain

$\circ$ Not sure at all $(25 \%)$

$\circ$ No information by which to judge $(0 \%)$

If you know, please list what type(s) of cancer she had? 


\section{Appendix B}

The Quick Coherence Technique helps you create a coherent state, offering access to your heart's intelligence. It uses the power of your heart to balance thoughts and emotion, helping you to achieve a neutral, poised state for clear thinking. It is a powerful technique that connects you with your energetic heart zone thelp you release stress, balance your emotions, and feel better fast.

Quick coherence will help you find a feeling of ease and inner harmony that will be reflected in your heart rhythms. The heart is a primary generator of rhythm in your body, influencing brain processes that control your nervous system, cognitive function and emotion. More coherent heart rhythms facilitate brain function, allowing you more access to your higher intelligence so you can improve your focus, creativity, intuition and higher-level decisionmaking. When you're in heart-rhythm coherence, you perform at your best - what athletes call being in the zone. You feel confident, positive, focused and calm yet energized.

Step One, "Heart Focus": Focus your attention on the area around your heart, the area in the center of your chest. If you prefer, the first couple of times you try it, place your hand over the center of your chest to help keep your attention in the heart area.

Step Two, "Heart Breathing": Breathe deeply but normally and feel as if your breath is coming in and going out through your heart are. As you inhale, feel as if your breath is flowing in through the heart, and as you exhale, feel it leaving through this area. Breathe slowly and casually, a little deeper than normal. Continue breathing with ease until you find a natural inner rhythm that feels good to you.

Step Three, "Heart Feeling": As you maintain your heart focus and heart breathing, activate a positive feeling. Recall a positive feeling, a time when you felt good inside, and try to re- 
experience the feeling. One of the easiest ways to generate positive, heart-based feeling is to remember a special place you've been to or the love you feel for a close friend or family member or treasured pet. This is the most important step. 
Appendix C

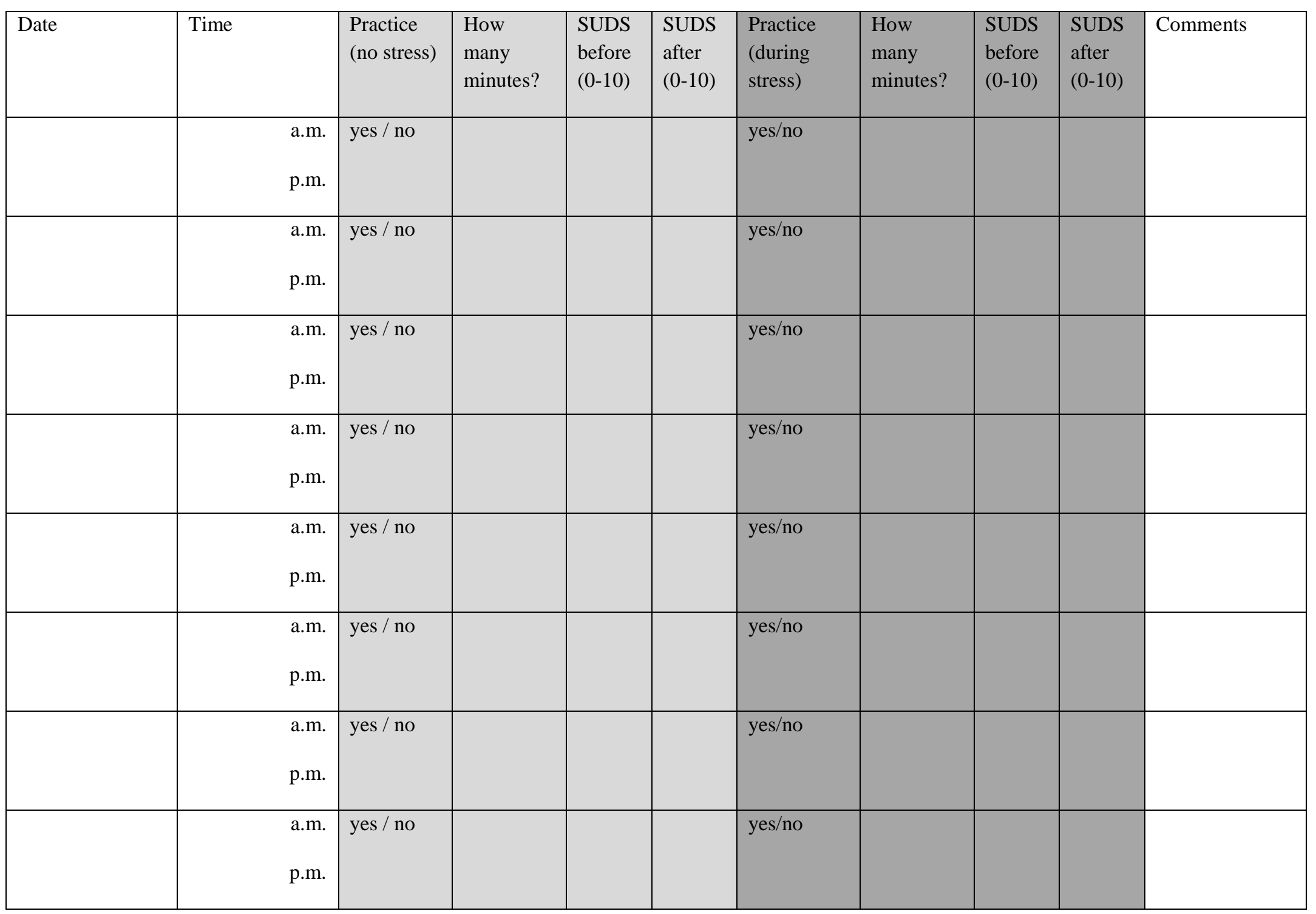




\section{Appendix D}

Assessed for eligibility $(n=325)$

\section{Enrollment}
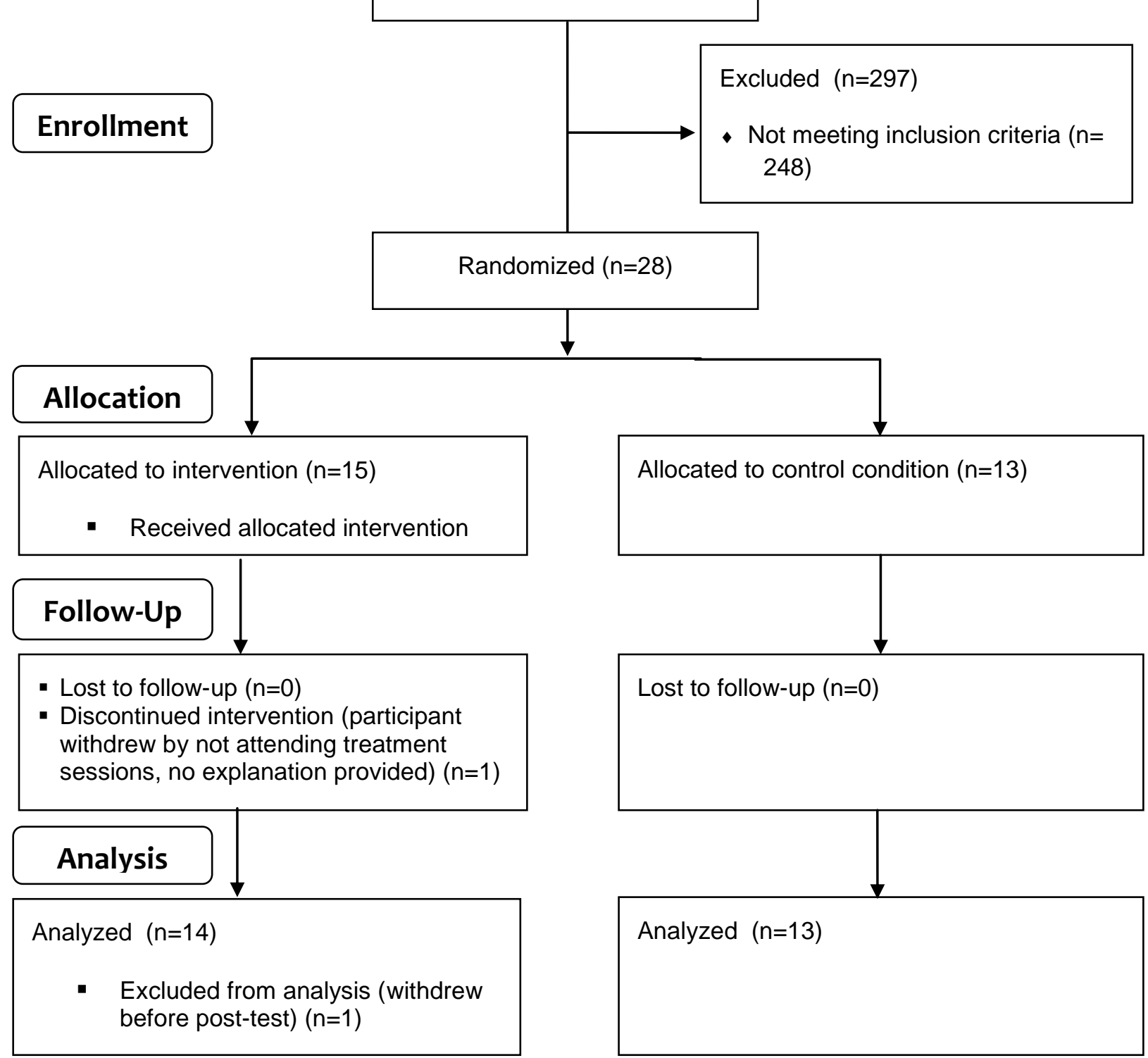

Figure 1. Summary of participant flow through the research protocol. 


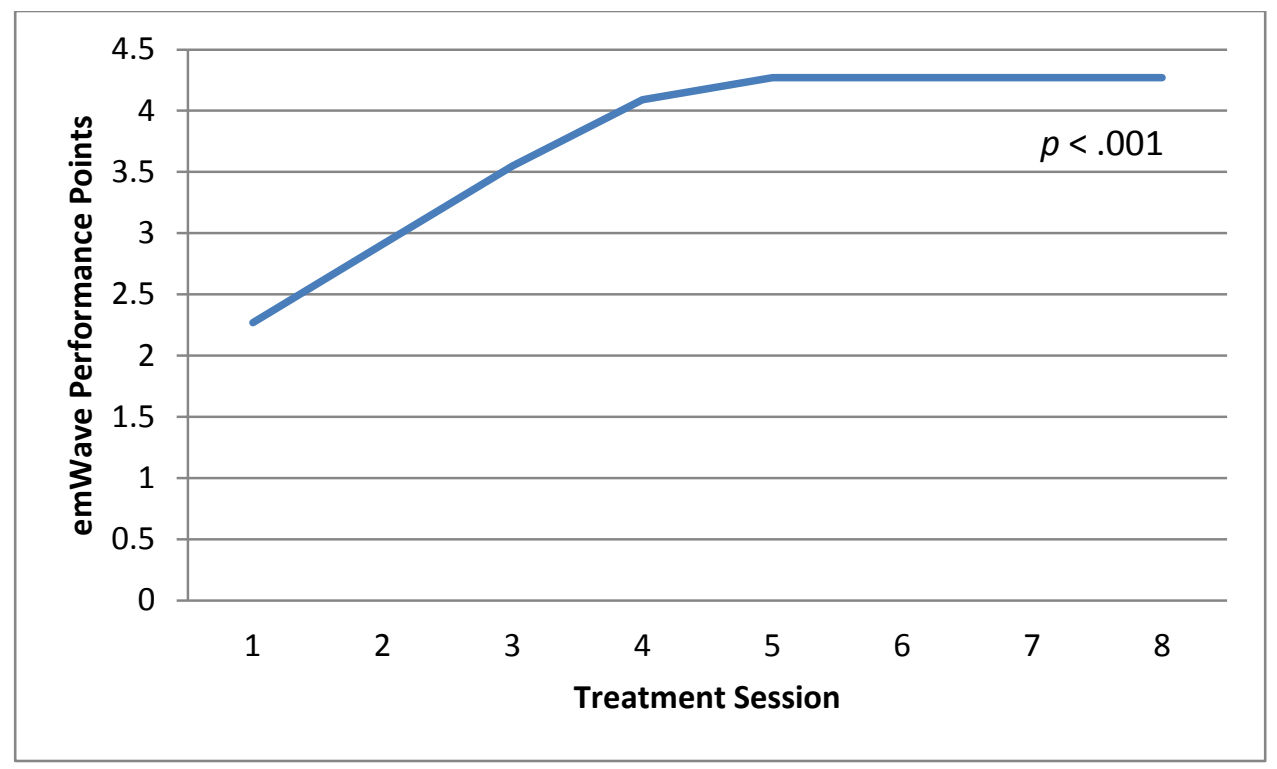

Figure 2. Repeated measures ANOVA for emWave performance points across session. Note: the last observation was carried forward for participants who completed only $<8$ training sessions; Session $1<$ Session $2<$ Session $3<$ Sessions 4-8. 


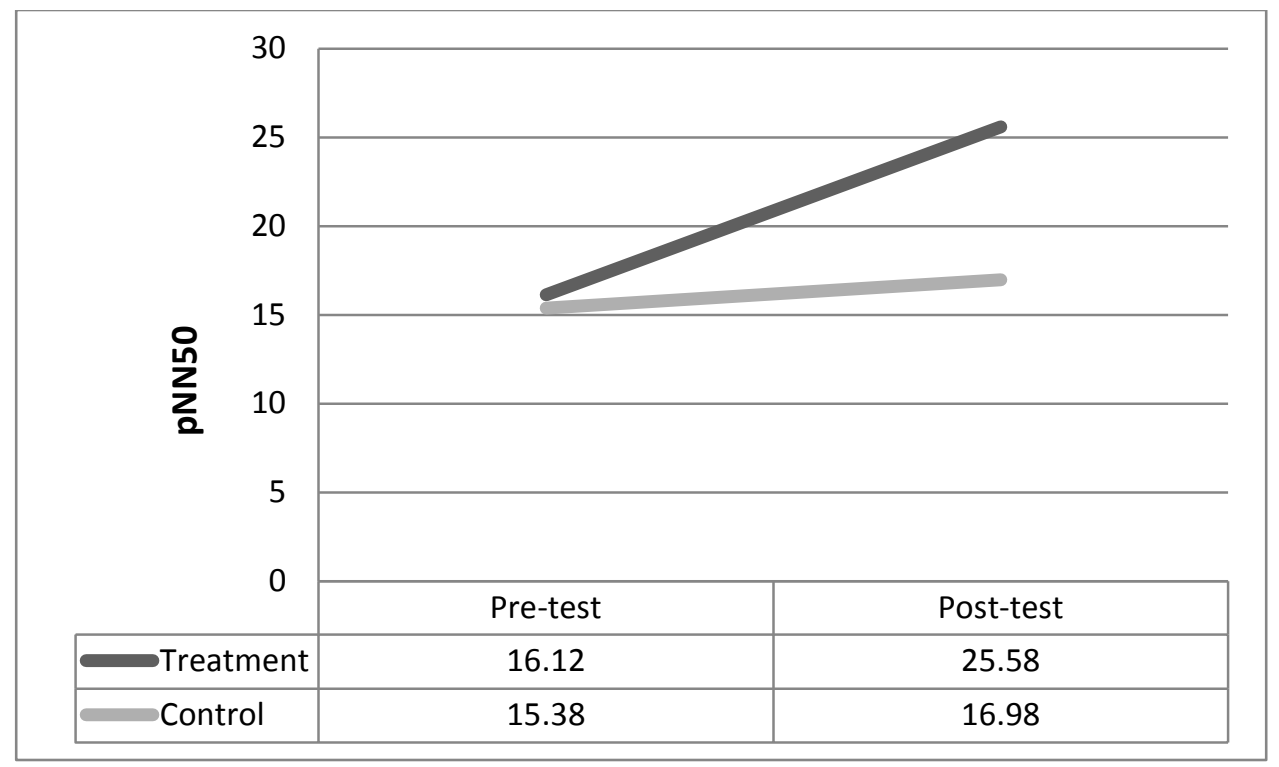

Figure 3. Interaction between Group and Session for pNN50 during stress, $p<.05$. 


\section{Appendix E}

Table 1. Summary of demographic information for study participants.

\begin{tabular}{|c|c|c|c|c|}
\hline & & Treatment & Control & Overall \\
\hline Age & Range & $18-30$ & $19-29$ & $18-30$ \\
\hline \multirow{2}{*}{ in years } & Mean & 22.29 & 23.15 & 22.54 \\
\hline & $S D$ & 3.6 & 4.06 & 3.82 \\
\hline Gender & Male & $2(14.3 \%)$ & $2(15.4 \%)$ & $4(14.8 \%)$ \\
\hline$N(\%)$ & Female & $12(85.7 \%)$ & $11(84.6 \%)$ & $23(85.2 \%)$ \\
\hline Race & White & $14(100 \%)$ & $11(84.6 \%)$ & $26(92.8 \%)$ \\
\hline \multirow[t]{2}{*}{$N(\%)$} & Black & 0 & $1(7.7 \%)$ & $1(3.6 \%)$ \\
\hline & Other & 0 & $1(7.7 \%)$ & $1(3.6 \%)$ \\
\hline Ethnicity & Non-Hispanic & $14(100 \%)$ & $11(84.6 \%)$ & $26(92.9 \%)$ \\
\hline$N(\%)$ & Hispanic & 0 & $2(15.4 \%)$ & $2(7.1 \%)$ \\
\hline \multirow{3}{*}{$\begin{array}{l}\text { Weight } \\
\text { in pounds }\end{array}$} & Range & $102-227.4$ & $96-239.4$ & $96-239.4$ \\
\hline & Mean & 146.83 & 156.19 & 151.5 \\
\hline & $S D$ & 34.93 & 39.42 & 36.07 \\
\hline \multirow{3}{*}{$\begin{array}{l}\text { Height } \\
\text { in inches }\end{array}$} & Range & $59-69$ & $58.75-73.75$ & $58.75-73.75$ \\
\hline & Mean & 64.59 & 65.4 & 64.96 \\
\hline & $S D$ & 3.01 & 4.48 & 3.67 \\
\hline \multirow[t]{3}{*}{ BMI } & Range & $18.21-37.13$ & $19.55-36.94$ & $18.21-37.13$ \\
\hline & Mean & 24.76 & 25.53 & 25.13 \\
\hline & $S D$ & 5.77 & 5.42 & 5.51 \\
\hline Parent income & $<24999$ & $1(7.1 \%)$ & $2(15.4 \%)$ & $3(11.1 \%)$ \\
\hline \multirow{7}{*}{$N(\%)$} & $25-34999$ & 0 & $2(15.4 \%)$ & $2(7.4 \%)$ \\
\hline & $35-49999$ & $3(21.4 \%)$ & $4(30.7 \%)$ & $7(25.9 \%)$ \\
\hline & $50-74999$ & $6(42.9 \%)$ & $1(7.7 \%)$ & $7(25.9 \%)$ \\
\hline & 75-99999 & $1(7.1 \%)$ & $2(15.4 \%)$ & $3(11.1 \%)$ \\
\hline & 100-149999 & 0 & $1(7.7 \%)$ & $1(3.6 \%)$ \\
\hline & $150+$ & $1(7.1 \%)$ & $1(7.7 \%)$ & $2(7.4 \%)$ \\
\hline & Missing & $2(14.4 \%)$ & 0 & $2(7.4 \%)$ \\
\hline Student Status & Undergraduate & $6(42.9 \%)$ & $6(46.2 \%)$ & $12(42.9 \%)$ \\
\hline$N(\%)$ & Graduate & $8(57.1 \%)$ & $7(53.8 \%)$ & $16(57.1 \%)$ \\
\hline Number of & 4 & $10(71.4 \%)$ & --- & --- \\
\hline Treatment & 5 & $2(14.3 \%)$ & & \\
\hline Sessions & 6 & 0 & & \\
\hline \multirow[t]{2}{*}{$N(\%)$} & 7 & 0 & & \\
\hline & 8 & $2(14.3 \%)$ & & \\
\hline
\end{tabular}

Note. Groups did not differ significantly on any demographic variable. 
Table 2. Correlations among Physiological Outcomes and BMI, Age, and Sex at Baseline.

\begin{tabular}{|c|c|c|c|}
\hline & BMI & Age & Sex \\
\hline \multicolumn{4}{|l|}{ LF HRV } \\
\hline Rest & -0.10 & 0.10 & 0.17 \\
\hline \multicolumn{4}{|c|}{ Mental Arithmetic } \\
\hline Reactivity & -0.06 & $0.41 *$ & -0.11 \\
\hline Recovery & -0.06 & 0.13 & 0.07 \\
\hline \multicolumn{4}{|l|}{ Handgrip } \\
\hline Reactivity & -0.10 & $0.41 *$ & -0.13 \\
\hline Recovery & -0.12 & 0.06 & 0.11 \\
\hline \multicolumn{4}{|l|}{ HF HRV } \\
\hline Rest & -0.17 & 0.21 & 0.34 \\
\hline \multicolumn{4}{|c|}{ Mental Arithmetic } \\
\hline Reactivity & -0.26 & 0.31 & 0.14 \\
\hline Recovery & -0.13 & 0.17 & 0.37 \\
\hline \multicolumn{4}{|l|}{ Handgrip } \\
\hline Reactivity & -0.18 & 0.30 & 0.01 \\
\hline Recovery & -0.24 & 0.20 & 0.32 \\
\hline \multicolumn{4}{|l|}{ SDNN } \\
\hline Rest & -0.15 & $0.40 *$ & 0.17 \\
\hline \multicolumn{4}{|c|}{ Mental Arithmetic } \\
\hline Reactivity & -0.22 & -0.33 & -0.01 \\
\hline Recovery & -0.15 & 0.19 & 0.26 \\
\hline \multicolumn{4}{|l|}{ Handgrip } \\
\hline Reactivity & -0.17 & $0.54 * *$ & 0.5 \\
\hline Recovery & -0.14 & 0.18 & 0.18 \\
\hline \multicolumn{4}{|l|}{ pNN50 } \\
\hline Rest & -0.25 & 0.33 & 0.31 \\
\hline \multicolumn{4}{|c|}{ Mental Arithmetic } \\
\hline Reactivity & -0.20 & $.40 *$ & 0.01 \\
\hline Recovery & -0.16 & 0.28 & 0.32 \\
\hline \multicolumn{4}{|l|}{ Handgrip } \\
\hline Reactivity & -0.15 & 0.33 & 0.20 \\
\hline Recovery & -0.35 & 0.31 & 0.24 \\
\hline \multicolumn{4}{|l|}{ RMSSD } \\
\hline Rest & -0.21 & 0.36 & 0.30 \\
\hline \multicolumn{4}{|c|}{ Mental Arithmetic } \\
\hline Reactivity & -0.21 & $0.41 *$ & 0.11 \\
\hline Recovery & -0.20 & 0.25 & 0.36 \\
\hline \multicolumn{4}{|l|}{ Handgrip } \\
\hline Reactivity & -0.15 & $0.43^{*}$ & 0.15 \\
\hline Recovery & -0.24 & 0.29 & 0.30 \\
\hline
\end{tabular}

$* p<.05 . * * p<.01$. 
Table 3. ANOVA for Evidence of Heart Rate Reactivity to Tasks.

\begin{tabular}{llllll}
\hline & & & \multicolumn{3}{c}{$\boldsymbol{F}$} \\
\cline { 4 - 6 } & Mean & $S D$ & Group & Period & Group X Period \\
\cline { 2 - 6 } $\begin{array}{l}\text { Pre-Treatment } \\
\quad \text { Rest }\end{array}$ & 77.85 & 9.98 & & & \\
$\quad$ Mental Arithmetic & 84.93 & 10.27 & $0.58^{\mathrm{ns}}$ & $62.00^{* * * *}$ & $0.72^{\mathrm{ns}}$ \\
$\quad$ Handgrip & 87.25 & 12.22 & $0.47^{\mathrm{ns}}$ & $34.01^{* * *}$ & $0.10^{\mathrm{ns}}$ \\
Post-treatment & & & & & \\
$\quad$ Rest & 76.51 & 12.36 & & & \\
$\quad$ Mental Arithmetic & 81.48 & 10.61 & $0.04^{\mathrm{ns}}$ & $37.14^{* * *}$ & $1.34^{\mathrm{ns}}$ \\
$\quad$ Handgrip & 83.84 & 12.81 & $0.41^{\mathrm{ns}}$ & $23.21^{* * *}$ & $0.03^{\mathrm{ns}}$ \\
\hline$* * * p<.001$. & & & & &
\end{tabular}


Table 4. ANCOVA for Evidence of Task Differences in Physiological Outcomes controlling for resting physiology and age.

\begin{tabular}{|c|c|c|c|c|c|}
\hline \multirow{3}{*}{ HF HRV } & \multirow{2}{*}{ EM Mean } & \multirow[b]{2}{*}{$S E$} & \multicolumn{3}{|c|}{$F$} \\
\hline & & & Group & Task & Group X Task \\
\hline & & & & & \\
\hline \multicolumn{3}{|l|}{ Pre-Treatment } & 0.03 & 1.34 & 1.10 \\
\hline Mental Arithmetic & 6.54 & 0.10 & & & \\
\hline Handgrip & 6.18 & 0.16 & & & \\
\hline \multicolumn{3}{|l|}{ Post-treatment } & 0.68 & 0.02 & 1.64 \\
\hline Mental Arithmetic & 6.71 & 0.11 & & & \\
\hline Handgrip & 6.41 & 0.20 & & & \\
\hline \multicolumn{6}{|l|}{ LF HRV } \\
\hline \multicolumn{3}{|l|}{ Pre-Treatment } & 2.94 & 1.92 & 1.10 \\
\hline Mental Arithmetic & 7.16 & 0.09 & & & \\
\hline Handgrip & 6.77 & 0.15 & & & \\
\hline \multicolumn{3}{|l|}{ Post-treatment } & $4.58^{*}$ & 0.86 & 3.41 \\
\hline Mental Arithmetic & 7.37 & 0.12 & & & \\
\hline Handgrip & 7.10 & 0.12 & & & \\
\hline \multicolumn{6}{|l|}{ SDNN } \\
\hline \multicolumn{3}{|l|}{ Pre-Treatment } & 0.56 & 4.05 & 0.03 \\
\hline Mental Arithmetic & 60.28 & 2.89 & & & \\
\hline Handgrip & 62.55 & 3.58 & & & \\
\hline \multicolumn{3}{|l|}{ Post-treatment } & 3.43 & 0.18 & 1.32 \\
\hline Mental Arithmetic & 65.67 & 2.64 & & & \\
\hline Handgrip & 68.56 & 3.36 & & & \\
\hline \multicolumn{6}{|l|}{ pNN50 } \\
\hline \multicolumn{3}{|l|}{ Pre-Treatment } & 0.08 & 0.44 & 0.05 \\
\hline Mental Arithmetic & 16.35 & 1.52 & & & \\
\hline Handgrip & 15.21 & 2.12 & & & \\
\hline \multicolumn{3}{|l|}{ Post-treatment } & $6.34 *$ & 0.04 & 2.72 \\
\hline Mental Arithmetic & 21.33 & 21.33 & & & \\
\hline Handgrip & 21.25 & 21.25 & & & \\
\hline \multicolumn{6}{|l|}{ RMSSD } \\
\hline \multicolumn{3}{|l|}{ Pre-Treatment } & 0.65 & 0.21 & 0.08 \\
\hline Mental Arithmetic & 37.28 & 1.61 & & & \\
\hline Handgrip & 37.58 & 2.75 & & & \\
\hline \multicolumn{3}{|l|}{ Post-treatment } & 0.45 & 3.05 & 4.04 \\
\hline Mental Arithmetic & 45.81 & 2.57 & & & \\
\hline Handgrip & 45.15 & 3.07 & & & \\
\hline
\end{tabular}


Table 5. Repeated Measures ANCOVAs on measures of HRV between first and final treatment sessions for treatment participants, controlling for age.

\begin{tabular}{llll}
\hline & $\begin{array}{l}\text { First } \\
\text { Session } \\
\text { (EMM, SE) }\end{array}$ & $\begin{array}{l}\text { Final } \\
\text { Session } \\
\text { (EMM, SE) }\end{array}$ & $\boldsymbol{F}$ \\
\cline { 3 - 4 } HF HRV & $7.00(0.22)$ & $6.61(0.22)$ & 0.00 \\
LF HRV & $7.88(2.5)$ & $8.58(0.25)$ & 0.78 \\
SDNN & $81.96(7.60)$ & $94.72(7.70)$ & 0.15 \\
pNN50 & $26.7(4.36)$ & $28.09(3.60)$ & 0.02 \\
RMSSD & $56.90(5.55)$ & $59.63(3.52)$ & 0.02 \\
\hline
\end{tabular}

Note: HF and LF values are natural log transformations of HRV data. 
Table 6. Repeated Measures ANOVA on emWave performance points across treatment sessions for treatment participants.

\begin{tabular}{|c|c|c|c|c|c|c|c|c|c|}
\hline & \multicolumn{9}{|l|}{ Session } \\
\hline & 1 & 2 & 3 & 4 & 5 & 6 & 7 & 8 & $F$ \\
\hline $\begin{array}{l}\text { emWave } \\
\text { Performance } \\
\text { Points }\end{array}$ & $\begin{array}{l}2.27^{\mathrm{a}, \mathrm{b}, \mathrm{c}, \mathrm{d}, \mathrm{e}} \\
(0.65)\end{array}$ & $\begin{array}{l}2.90^{\mathrm{a}, \mathrm{b}, \mathrm{c}, \mathrm{d}, \mathrm{e}} \\
(0.94)\end{array}$ & $\begin{array}{l}3.55^{\mathrm{a}, \mathrm{b}, \mathrm{c}, \mathrm{d}, \mathrm{e}} \\
(1.21)\end{array}$ & $\begin{array}{l}4.09^{\mathrm{a}} \\
(1.14)\end{array}$ & $\begin{array}{l}4.27^{\mathrm{b}} \\
(0.90)\end{array}$ & $\begin{array}{l}4.27^{\mathrm{c}} \\
(0.90)\end{array}$ & $\begin{array}{l}4.27^{\mathrm{d}} \\
(0.90)\end{array}$ & $\begin{array}{l}4.27^{\mathrm{e}} \\
(0.90)\end{array}$ & $32.90 * * *$ \\
\hline
\end{tabular}

$* * * p<.001$.

Note: Means represent last observation carried forward for participants completing less than 8 sessions. 
Table 7. ANCOVA for effects of Group and Session on tonic measures of HRV, controlling for age.

\begin{tabular}{|c|c|c|c|c|c|c|c|}
\hline & \multicolumn{2}{|c|}{ Pre-treatment } & \multicolumn{2}{|c|}{ Post-treatment } & \multicolumn{3}{|c|}{$F$} \\
\hline & EM Mean & $S E$ & EM Mean & $S E$ & Group & Session & $\begin{array}{l}\text { Group X } \\
\text { Session }\end{array}$ \\
\hline HF HRV & & & & & $3.02^{\mathrm{ns}}$ & $0.00^{\mathrm{ns}}$ & $0.02^{\mathrm{ns}}$ \\
\hline Treatment & 6.64 & 0.29 & 6.86 & 0.28 & & & \\
\hline Control & 6.13 & 0.30 & 6.28 & 0.29 & & & \\
\hline LF HRV & & & & & $7.10^{*}$ & $0.18^{\mathrm{ns}}$ & $0.00^{\mathrm{ns}}$ \\
\hline Treatment & 7.13 & 0.23 & 7.28 & 0.28 & & & \\
\hline Control & 6.33 & 0.24 & 6.48 & 0.29 & & & \\
\hline SDNN & & & & & $9.29^{* *}$ & $0.44^{\mathrm{ns}}$ & $0.38^{\mathrm{ns}}$ \\
\hline Treatment & 70.33 & 4.32 & 68.84 & 6.03 & & & \\
\hline Control & 49.16 & 4.49 & 53.39 & 6.25 & & & \\
\hline pNN50 & & & & & $0.71^{\mathrm{ns}}$ & $0.06^{\mathrm{ns}}$ & $1.83^{\mathrm{ns}}$ \\
\hline Treatment & 25.86 & 4.42 & 23.38 & 5.21 & & & \\
\hline Control & 15.85 & 4.58 & 23.52 & 5.41 & & & \\
\hline RMSSD & & & & & $1.48^{\mathrm{ns}}$ & $0.01^{\mathrm{ns}}$ & $0.08^{\mathrm{ns}}$ \\
\hline Treatment & 48.30 & 4.82 & 47.76 & 6.19 & & & \\
\hline Control & 35.14 & 5.00 & 44.67 & 6.42 & & & \\
\hline
\end{tabular}

$* p<.05 . * * p<.01$. 
Table 8. Group X Session ANCOVAs on HRV measures during stress, controlling for resting HRV and participant age.

\begin{tabular}{|c|c|c|c|c|c|c|c|}
\hline & Pre-test & & Post-test & & & $F$ & \\
\hline & EM Mean & $S E$ & EM Mean & $S E$ & Group & Session & $\begin{array}{l}\text { Group X } \\
\text { Session }\end{array}$ \\
\hline HF HRV & & & & & $0.16^{\mathrm{ns}}$ & $1.07^{\mathrm{ns}}$ & $2.09^{\mathrm{ns}}$ \\
\hline Treatment & 6.38 & 0.14 & 6.45 & 0.20 & & & \\
\hline Control & 6.34 & 0.14 & 6.26 & 0.20 & & & \\
\hline LF HRV & & & & & $3.18^{\mathrm{ns}}$ & $4.11^{\mathrm{ns}}$ & $3.80^{\mathrm{ns}}$ \\
\hline Treatment & 7.15 & 0.14 & 7.47 & 0.15 & & & \\
\hline Control & 6.78 & 0.15 & 7.00 & 0.16 & & & \\
\hline SDNN & & & & & $0.70^{\mathrm{ns}}$ & $3.38^{\mathrm{ns}}$ & $3.24^{\mathrm{ns}}$ \\
\hline Treatment & 64.12 & 4.29 & 72.13 & 4.04 & & & \\
\hline Control & 58.82 & 4.48 & 63.27 & 4.21 & & & \\
\hline pNN50 & & & & & $2.51^{\mathrm{ns}}$ & $9.65^{* *}$ & $7.43^{*}$ \\
\hline Treatment & 16.12 & 2.03 & 25.58 & 2.38 & & & \\
\hline Control & 15.38 & 2.11 & 16.98 & 2.47 & & & \\
\hline RMSSD & & & & & $1.84^{\mathrm{ns}}$ & $6.35^{*}$ & $3.58^{\mathrm{ns}}$ \\
\hline Treatment & 38.34 & 2.65 & 50.11 & 3.31 & & & \\
\hline Control & 36.52 & 2.76 & 40.85 & 3.43 & & & \\
\hline
\end{tabular}

${ }^{*} p<.05 . * * p<.01$. 
Table 9. Group X Session ANCOVAs on measures of HRV during recovery from stress, controlling for resting $H R V$ and age.

\begin{tabular}{|c|c|c|c|c|c|c|c|}
\hline & Pre-test & & Post-test & & & $F$ & \\
\hline & EM Mean & $S E$ & EM Mean & $S E$ & Group & Session & $\begin{array}{l}\text { Group X } \\
\text { Session }\end{array}$ \\
\hline HF HRV & & & & & $2.77^{\mathrm{ns}}$ & $2.38^{\mathrm{ns}}$ & $0.30^{\mathrm{ns}}$ \\
\hline Treatment & 6.89 & 0.13 & 7.00 & 0.17 & & & \\
\hline Control & 6.58 & 0.13 & 6.66 & 0.18 & & & \\
\hline LF HRV & & & & & $3.36^{\mathrm{ns}}$ & $0.01^{\mathrm{ns}}$ & $1.58^{\mathrm{ns}}$ \\
\hline Treatment & 7.41 & 0.13 & 7.45 & 0.20 & & & \\
\hline Control & 6.89 & 0.14 & 7.04 & 0.20 & & & \\
\hline SDNN & & & & & $0.31^{\mathrm{ns}}$ & $0.48^{\mathrm{ns}}$ & $1.86^{\mathrm{ns}}$ \\
\hline Treatment & 75.90 & 4.33 & 72.66 & 4.13 & & & \\
\hline Control & 65.28 & 4.51 & 74.87 & 4.29 & & & \\
\hline pNN50 & & & & & $3.08^{\mathrm{ns}}$ & $0.04^{\mathrm{ns}}$ & $0.01^{\mathrm{ns}}$ \\
\hline Treatment & 26.99 & 2.01 & 29.61 & 2.05 & & & \\
\hline Control & 23.10 & 2.10 & 25.64 & 2.13 & & & \\
\hline RMSSD & & & & & $2.36^{\mathrm{ns}}$ & $0.05^{\mathrm{ns}}$ & $0.06^{\mathrm{ns}}$ \\
\hline Treatment & 51.91 & 3.77 & 55.16 & 2.94 & & & \\
\hline Control & 46.40 & 3.93 & 49.79 & 3.05 & & & \\
\hline
\end{tabular}

$* p<.05$

Note: HF and LF values are natural log transformations of HRV data. 
Table 10. Exploratory Group X Session ANCOVAs on HRV measures during stress, controlling for resting $H R V$ and participant age, and after excluding select treatment participants.

\begin{tabular}{|c|c|c|c|c|c|c|c|}
\hline & Pre-test & & Post-test & & $F$ & & \\
\hline & EM Mean & $S E$ & EM Mean & $S E$ & Group & Session & $\begin{array}{l}\text { Group X } \\
\text { Session }\end{array}$ \\
\hline HF HRV & & & & & $1.63^{\mathrm{ns}}$ & $1.67^{\mathrm{ns}}$ & $2.83^{\mathrm{ns}}$ \\
\hline Treatment & 6.38 & 0.14 & 6.67 & 0.18 & & & \\
\hline Control & 6.34 & 0.14 & 6.45 & 0.19 & & & \\
\hline LF HRV & & & & & $5.24 *$ & $5.36 *$ & $5.01 *$ \\
\hline Treatment & 7.15 & 0.14 & 7.48 & 0.15 & & & \\
\hline Control & 6.78 & 0.15 & 7.00 & 0.16 & & & \\
\hline SDNN & & & & & $1.67^{\mathrm{ns}}$ & $5.00 *$ & $5.01 *$ \\
\hline Treatment & 64.12 & 4.29 & 72.13 & 4.04 & & & \\
\hline Control & 58.82 & 4.48 & 63.27 & 4.21 & & & \\
\hline pNN50 & & & & & $2.76^{\mathrm{ns}}$ & $14.50 * *$ & $11.64 * *$ \\
\hline Treatment & 16.12 & 2.03 & 25.58 & 2.38 & & & \\
\hline Control & 15.38 & 2.11 & 16.98 & 2.47 & & & \\
\hline RMSSD & & & & & $3.09^{\mathrm{ns}}$ & $9.46 * *$ & $6.20 *$ \\
\hline Treatment & 38.34 & 2.65 & 50.11 & 3.31 & & & \\
\hline Control & 36.52 & 2.76 & 40.85 & 3.43 & & & \\
\hline
\end{tabular}

$*_{p}<.05 . * * p<.01$. 
Table 11. Group X Session ANOVAs on self-report measures of affective response, stress, and psychological symptom distress.

\begin{tabular}{|c|c|c|c|c|c|c|}
\hline & & & & Group & Session & $\begin{array}{l}\text { Group X } \\
\text { Session }\end{array}$ \\
\hline & & \multicolumn{2}{|l|}{ Mean (SD) } & \multicolumn{3}{|c|}{$F$} \\
\hline \multicolumn{7}{|c|}{ MAACLR Anxiety } \\
\hline Baseline & $\begin{array}{l}\text { Treatment } \\
\text { Control }\end{array}$ & $\begin{array}{l}1.93(2.56) \\
0.77(1.24)\end{array}$ & $\begin{array}{l}0.71(1.07) \\
0.38(0.51)\end{array}$ & 2.51 & $4.61 *$ & 1.24 \\
\hline Task & $\begin{array}{l}\text { Treatment } \\
\text { Control }\end{array}$ & $\begin{array}{l}2.89(1.69) \\
2.38(1.19)\end{array}$ & $\begin{array}{l}1.61(1.06) \\
2.08(1.08)\end{array}$ & 0.40 & 4.18 & 1.99 \\
\hline \multicolumn{7}{|c|}{ MAACLR Depression } \\
\hline Baseline & $\begin{array}{l}\text { Treatment } \\
\text { Control }\end{array}$ & $\begin{array}{l}0.86(1.35) \\
0.69(1.03)\end{array}$ & $\begin{array}{l}0.50(1.02) \\
0.23(0.60)\end{array}$ & 0.34 & $7.82 * *$ & 0.13 \\
\hline Task & $\begin{array}{l}\text { Treatment } \\
\text { Control }\end{array}$ & $\begin{array}{l}1.00(1.29) \\
1.19(1.20)\end{array}$ & $\begin{array}{l}0.71(0.96) \\
1.38(1.18)\end{array}$ & 2.25 & 0.35 & 1.51 \\
\hline \multicolumn{7}{|c|}{ MAACLR Hostility } \\
\hline Baseline & $\begin{array}{l}\text { Treatment } \\
\text { Control }\end{array}$ & $\begin{array}{l}0.36(1.08) \\
0.00(0.00)\end{array}$ & $\begin{array}{l}0.29(0.73) \\
0.80(0.28)\end{array}$ & 1.51 & 0.15 & 0.26 \\
\hline Task & $\begin{array}{l}\text { Treatment } \\
\text { Control }\end{array}$ & $\begin{array}{l}2.12(1.69) \\
1.88(1.70)\end{array}$ & $\begin{array}{l}1.92(2.34) \\
1.81(1.38)\end{array}$ & 0.01 & 1.26 & 1.01 \\
\hline \multicolumn{7}{|c|}{ MAACLR Positive Affect } \\
\hline Baseline & $\begin{array}{l}\text { Treatment } \\
\text { Control }\end{array}$ & $\begin{array}{l}4.57(2.65) \\
6.15(5.80)\end{array}$ & $\begin{array}{l}3.71(2.70) \\
6.46(4.37)\end{array}$ & 0.91 & 0.01 & 3.19 \\
\hline Task & $\begin{array}{l}\text { Treatment } \\
\text { Control }\end{array}$ & $\begin{array}{l}0.73(1.36) \\
1.01(1.14)\end{array}$ & $\begin{array}{l}1.19(1.84) \\
1.23(1.13)\end{array}$ & 0.26 & 1.10 & 0.65 \\
\hline MAACLR & sation Seeking & & & & & \\
\hline Baseline & $\begin{array}{l}\text { Treatment } \\
\text { Control }\end{array}$ & $\begin{array}{l}0.43(0.65) \\
0.58(1.44)\end{array}$ & $\begin{array}{l}0.21(0.43) \\
0.46(6.6)\end{array}$ & 0.34 & 0.16 & 1.12 \\
\hline Arithmetic & $\begin{array}{l}\text { Treatment } \\
\text { Control }\end{array}$ & $\begin{array}{l}0.64(1.08) \\
0.15(0.38)\end{array}$ & $\begin{array}{l}0.79(0.89) \\
0.46(0.66)\end{array}$ & 2.82 & $6.44^{*}$ & 0.04 \\
\hline Handgrip & $\begin{array}{l}\text { Treatment } \\
\text { Control }\end{array}$ & $\begin{array}{l}1.07(1.14) \\
1.23(1.42)\end{array}$ & $\begin{array}{l}0.93(0.83) \\
0.85(0.90)\end{array}$ & 0.00 & 0.72 & 0.06 \\
\hline PSS & $\begin{array}{l}\text { Treatment } \\
\text { Control }\end{array}$ & $\begin{array}{l}40.21(9.33) \\
37.49(6.40)\end{array}$ & $\begin{array}{l}34.71(8.99) \\
36.08(5.82)\end{array}$ & 0.06 & $6.23 *$ & 2.18 \\
\hline
\end{tabular}


Table 11. (continued)

\begin{tabular}{|c|c|c|c|c|c|c|}
\hline & & & & Group & Session & $\begin{array}{l}\text { Group X } \\
\text { Session }\end{array}$ \\
\hline & & \multicolumn{2}{|l|}{ Mean (SD) } & \multicolumn{3}{|c|}{$F$} \\
\hline & & Pre-Test & Post-Test & & & \\
\hline \multicolumn{7}{|l|}{ BSI } \\
\hline \multirow[t]{2}{*}{ Somatization } & Treatment & $0.66(0.58)$ & $0.39(0.63)$ & 0.09 & $5.24 *$ & 0.54 \\
\hline & Control & $0.54(0.58)$ & $0.40(0.49)$ & & & \\
\hline Obsessive- & Treatment & $1.73(1.05)$ & $1.24(1.15)$ & 0.61 & 3.04 & 1.61 \\
\hline Compulsive & Control & $1.27(0.73)$ & $1.19(0.69)$ & & & \\
\hline \multirow{2}{*}{ Depression } & Treatment & $0.68(0.70)$ & $0.45(0.69)$ & 0.10 & 3.72 & 0.03 \\
\hline & Control & $0.77(0.79)$ & $0.50(0.45)$ & & & \\
\hline \multirow[t]{2}{*}{ Anxiety } & Treatment & $1.17(0.80)$ & $0.64(0.79)$ & $4.28 *$ & $5.44 *$ & 2.81 \\
\hline & Control & $0.55(0.48)$ & $0.45(0.32)$ & & & \\
\hline \multirow[t]{2}{*}{ Hostility } & Treatment & $0.63(0.52)$ & $0.51(0.44)$ & 0.30 & 2.23 & 0.03 \\
\hline & Control & $0.52(0.51)$ & $0.43(0.45)$ & & & \\
\hline \multirow[t]{2}{*}{ Phobic Anxiety } & Treatment & $0.29(0.41)$ & $0.24(0.68)$ & 0.75 & 0.48 & 0.48 \\
\hline & Control & $0.15(0.45)$ & $0.12(0.24)$ & & & \\
\hline Paranoid & Treatment & $0.51(0.61)$ & $0.44(0.52)$ & 0.14 & 2.69 & 0.94 \\
\hline Ideation & Control & $0.55(0.49)$ & $0.28(0.41)$ & & & \\
\hline \multirow[t]{2}{*}{ Psychoticism } & Treatment & $0.45(0.52)$ & $0.36(0.54)$ & 0.00 & 3.01 & 0.06 \\
\hline & Control & $0.46(0.45)$ & $0.34(0.30)$ & & & \\
\hline Interpersonal & Treatment & $0.75(0.80)$ & $0.77(0.93)$ & 0.10 & 0.54 & 0.78 \\
\hline Sensitivity & Control & $0.77(0.73)$ & $0.58(0.62)$ & & & \\
\hline \multirow[t]{2}{*}{ Additional Items } & Treatment & $0.93(0.65)$ & $0.61(0.82)$ & 0.08 & 2.12 & 1.67 \\
\hline & Control & $0.71(0.64)$ & $0.69(0.63)$ & & & \\
\hline Global Severity & Treatment & $0.80(0.54)$ & $0.57(0.62)$ & 0.43 & $5.51 *$ & 0.38 \\
\hline Index & Control & $0.64(0.42)$ & $0.50(0.32)$ & & & \\
\hline
\end{tabular}

$* p<.05 . * * p<.01$.

Note. No significant interactions between session and group observed (all $p \mathrm{~s}>.05$ ). 